\title{
Genes involved in thoracic exoskeleton formation during the pupal-to-adult molt in a social insect model, Apis mellifera
}

Michelle Prioli Miranda Soares', Angel Roberto Barchuk ${ }^{2}$, Ana Carolina Quirino Simões ${ }^{3}$, Alexandre dos Santos Cristino ${ }^{4}$, Flávia Cristina de Paula Freitas ${ }^{1}$, Luísa Lange Canhos ${ }^{5}$ and Márcia Maria Gentile Bitondi ${ }^{5^{*}}$

\begin{abstract}
Background: The insect exoskeleton provides shape, waterproofing, and locomotion via attached somatic muscles. The exoskeleton is renewed during molting, a process regulated by ecdysteroid hormones. The holometabolous pupa transforms into an adult during the imaginal molt, when the epidermis synthe3sizes the definitive exoskeleton that then differentiates progressively. An important issue in insect development concerns how the exoskeletal regions are constructed to provide their morphological, physiological and mechanical functions. We used whole-genome oligonucleotide microarrays to screen for genes involved in exoskeletal formation in the honeybee thoracic dorsum. Our analysis included three sampling times during the pupal-to-adult molt, i.e., before, during and after the ecdysteroid-induced apolysis that triggers synthesis of the adult exoskeleton.
\end{abstract}

Results: Gene ontology annotation based on orthologous relationships with Drosophila melanogaster genes placed the honeybee differentially expressed genes (DEGs) into distinct categories of Biological Process and Molecular Function, depending on developmental time, revealing the functional elements required for adult exoskeleton formation. Of the 1,253 unique DEGs, 547 were upregulated in the thoracic dorsum after apolysis, suggesting induction by the ecdysteroid pulse. The upregulated gene set included 20 of the 47 cuticular protein (CP) genes that were previously identified in the honeybee genome, and three novel putative CP genes that do not belong to a known CP family. In situ hybridization showed that two of the novel genes were abundantly expressed in the epidermis during adult exoskeleton formation, strongly implicating them as genuine CP genes. Conserved sequence motifs identified the CP genes as members of the CPR, Tweedle, Apidermin, CPF, CPLCP1 and Analogous-to-Peritrophins families. Furthermore, 28 of the 36 muscle-related DEGs were upregulated during the de novo formation of striated fibers attached to the exoskeleton. A search for cis-regulatory motifs in the $5^{\prime}$-untranslated region of the DEGs revealed potential binding sites for known transcription factors. Construction of a regulatory network showed that various upregulated CP- and muscle-related genes (15 and 21 genes, respectively) share common elements, suggesting co-regulation during thoracic exoskeleton formation.

Conclusions: These findings help reveal molecular aspects of rigid thoracic exoskeleton formation during the ecdysteroid-coordinated pupal-to-adult molt in the honeybee.

Keywords: Cuticular protein genes, Metamorphosis, Molt, Thoracic musculature, Microarrays, Honeybee, Apis mellifera

\footnotetext{
* Correspondence: mmgbit@usp.br

${ }^{5}$ Departamento de Biologia, Faculdade de Filosofia, Ciências e Letras de Ribeirão Preto, Universidade de São Paulo, Ribeirão Preto, SP, Brasil Full list of author information is available at the end of the article
} 


\section{Background}

Insect development occurs through a series of exoskeleton (cuticle) renewals, or molts, that are timed by ecdysteroids pulses. Molt succession includes apolysis (the separation of the cuticle from the epidermis), synthesis of a new cuticle and ecdysis (the shedding of the cuticle of the preceding instar, or stage).

After four molting episodes, the honeybee larva reaches the fifth larval instar without phenotypic changes, except for a considerable increase in size. The larva-to-pupa metamorphic molt takes place within the cuticle of the fifth larval instar. This is followed by pupal ecdysis, after which a white pupa breaks free from the larval cuticle. A genuine honeybee pupa exists for a relatively short period, lasting approximately $40 \mathrm{~h}$ from pupal ecdysis. Apolysis marks the onset of adult cuticle synthesis and deposition. During the next $160 \mathrm{~h}$, the bee is a pharate-adult, meaning that it is producing the adult cuticle underneath the pupal cuticle. Melanization starts and intensifies in the adult cuticle throughout the final half of the pharate-adult period, even after adult ecdysis. Concomitantly, the adult cuticle becomes increasingly sclerotized [1-4]. Thus, development toward the adult stage involves the reconstruction and maturation of the definitive cuticular exoskeleton, formation of internal tissues and organs, and programmed cell death of many larval tissues.

The structure, chemical composition, mechanical properties and renewal of the insect exoskeleton during growth and metamorphosis have been extensively studied $[5,6]$. The exoskeleton mainly consists of the polysaccharide chitin and a variety of structural proteins, the CPs. Nearly a decade ago, Willis et al., [7] compiled 139 insect CPs derived from direct sequencing of purified proteins, or from conceptual translations of DNA sequences. Since then, insect genome projects have expanded the number and types of potential CPs, thus underscoring the variety of cuticleforming proteins [8]. However, except for extensive studies on cuticular gene expression in Anopheles gambiae [9-11], few putative $\mathrm{CP}$ genes in annotated insect genomes have been experimentally validated (by determining transcript tissue-specificity or developmental profiles). In A. mellifera, only six of the $47 \mathrm{CP}$ genes screened in the annotated genome [8] have been validated, including the AmelCPR14 gene [12] (bearing the chitin-binding R\&R Consensus [13]), three genes in the Apidermins class [14], and the Tweedle class genes, AmelTwdl1 and AmelTwdl2 [15].

Thoracic exoskeleton construction and differentiation in pharate-adults occur concomitantly with the de novo formation of striated muscle fibers. The larval thoracic muscles are entirely disintegrated during the honeybee metamorphic molt, and are replaced by imaginal muscles originating from myoblast precursors, which elongate, join, and form the striated muscle fibers. Motor function is accomplished by the attachment of these muscle fibers to specific points, or ingrowths, in the developing thoracic exoskeleton [1]. Thus, the integument (epidermis and cuticular exoskeleton) and associated musculature form a cohesive and functional structure.

The morphological and cellular changes in the dorsal portion of the honeybee thorax during the pupal-toadult molt were previously characterized in our laboratory using conventional light microscopy [4] and transmission electron microscopy [16]. In addition to these detailed morphological descriptions, we used oligonucleotide microarray hybridization analysis, real-time RT-PCR (RT-qPCR) transcript quantification and fluorescent in situ hybridization to extend our knowledge. Unlike recent approaches in other insects, including microarray-based studies [17-19], we studied honeybee gene expression in the context of reconstruction of the hard exoskeleton that forms the thoracic dorsum, which includes the pronotum, mesonotum, metanotum, propodeal tergum (the dorsal part of the first abdominal segment that, in bees and wasps, becomes part of the adult thorax [1]) and the subjacent musculature. In contrast to the diversity of cuticle types that covers the entire insect body (hard/flexible; dark/clear), the thoracic dorsum is uniformly hardened and darkened. Thus, genes involved in the formation of a hard cuticle could be characterized. The thorax also has fewer glands that could contribute to the total RNA pool. Although there are integument glands in the thorax [20-22], they are much less abundant than in the abdomen. As described in Drosophila, the thoracic dorsum (notum) and associated musculature both originate from the same embryonic precursors (the wing imaginal discs) [23]. Thus we also investigated their potential shared gene regulatory networks for coordinated development.

Our microarray analyses identified genes involved in adult thoracic dorsum formation. We used this information to characterize potential binding sites for known transcription factors in the upstream control region (UCR) of the differentially expressed genes (DEGs). $\mathrm{CP}$ genes and muscle-related genes involved in thoracic dorsum formation were used to construct a putative gene regulatory network. Ecdysteroids control the pace of pupal-to-adult molt and adult thoracic dorsum development; therefore, we could also identify $\mathrm{CP}$ - and muscle-related genes that are potentially regulated by these hormones.

\section{Methods}

\section{Africanized Apis mellifera samples}

Newly-ecdysed pupae (white-eyed/unpigmented cuticle, Pw phase), pupae-in-apolysis (pink-eyed/unpigmented cuticle, Pp phase) and pharate-adults (brown-eyed/unpigmented cuticle, $\mathrm{Pb}$ phase; brown-eyed/light pigmented cuticle, Pbl phase) were collected from colonies maintained at an Experimental Apiary (University of São Paulo, 
Ribeirão Preto, SP, Brazil) and staged according to the criteria established by Michelette and Soares [3]. Dissections of the dorsal portion of the thorax, including pronotum, mesonotum, metanotum and the propodeal tergum, were performed in Ringer saline $(0.17 \mathrm{M} \mathrm{NaCl}$; $0.01 \mathrm{M} \mathrm{KCl} ; 0.003 \mathrm{M} \mathrm{CaCl}_{2}$ ). The total RNA was extracted from samples using $1 \mathrm{~mL}$ Trizol (Invitrogen, Life Technologies, cat. 15596-026) following manufacturer's instructions. The A. mellifera experiments performed in the present study comply with the current laws of Brazil.

\section{Semithin sections}

The thoracic dorsum of pupae and pharate-adults were dissected and immediately fixed in a mixture of glutaraldehyde $2 \%$ and paraformaldehyde $2 \%$ in $0.1 \mathrm{M}$ cacodylate buffer, $\mathrm{pH} 7.2$ for $2 \mathrm{~h}$ at $4^{\circ} \mathrm{C}$. Next, they were washed in $0.1 \mathrm{M}$ phosphate-buffered saline, $\mathrm{pH} 7.2$ (PBS) and re-fixed for $30 \mathrm{~min}$ in $1 \%$ osmium tetroxide in $0.1 \mathrm{M}$ PBS. The samples were then dehydrated in a graded acetone series and propylene oxide, pre-embedded in LX112 resin (Leica) in propylene oxide (1:1) and, finally, embedded in pure LX112 resin. Semithin sections $(0.5 \mu \mathrm{m})$ where stained with toluidine blue.

\section{Microarray hybridization and analysis}

Oligonucleotide-based microarrays (BeeOligo121106) were purchased from the Keck Center for Comparative and Functional Genomics, University of Illinois, UrbanaChampaign, USA. The array contains oligonucleotide probes including 12,915 protein-coding gene predictions from the honeybee genome, and probes specific for ESTs, pathogens and parasites in a total of 14,400 oligos, comprising each 36 nucleotides double-spotted on glass microscope slides [http://www.ebi.ac.uk/aerep/result?queryFor= PhysicalArrayDesign\&aAccession=A-MEXP-755].

The experiments were designed and performed to meet the Minimum Information About a Microarray Experiment (MIAME) specifications. The obtained microarray data were deposited at the Gene Expression Omnibus database [GEO:GSE43047]. The microarray experiments compared gene expression in the thoracic dorsum (including the pronotum, mesonotum, metanotum and propodeal tergum) of newly-ecdysed pupae, pupae-in-apolysis and pharate-adults ( $\mathrm{Pbl}$ phase) collected at the same time from a single honeybee colony. Two separate pools of 10 thoraces (only the dorsal portion) were prepared for each of these developmental phases. Total RNA was extracted from the pooled samples using Trizol reagent and following manufacturer's protocol. RNeasy Mini Kit, QIAGEN, cat. 74104, was used to purify the extracted RNA, which was quantified in Nanodrop (ND 1000). One microgram of the purified total RNA was used for RNA amplification with Amino AllylMessageAmp ${ }^{\text {tw }}$ II aRNA Amplification Kit (Ambion), and following manufacturer's protocol.
The microarray slides were UV cross-linked and subsequently incubated in $0.2 \%$ SDS for 2 min and rapidly in water and isopropanol, and centrifuged for $3 \mathrm{~min}$ at a low speed. Pre-hybridizations were performed in a mixture of $20 \mathrm{~mL}$ formamide, $33.2 \mathrm{~mL}$ SSC $20 \mathrm{x}, 10 \mathrm{~mL}$ Denhardt's solution 50x, $0.5 \mathrm{~mL}$ tRNA $10 \mathrm{mg} / \mathrm{mL}, 1 \mathrm{~mL}$ SDS $10 \%$ and $34.4 \mathrm{~mL}$ Milli-Q water for $60 \mathrm{~min}$ at $42^{\circ} \mathrm{C}$. The slides were then rinsed twice in Milli-Q water, soaked in isopropyl alcohol and dried by centrifugation at low speed for $3 \mathrm{~min}$.

Twenty micrograms of the amplified RNA samples (aRNA) were used for dual channel microarray hybridization with $\mathrm{Cy} 3$ and $\mathrm{Cy} 5$ dyes (dye swap). Dye swaps were done for each comparison and two slides were used to evaluate the differential expression. The RNA was dyecoupled and combined with hybridization buffer (49\% Formamide, 49\% SSC 20x and 0.2\% SDS), pre-heated at $55^{\circ} \mathrm{C}$ for $3 \mathrm{~min}$, applied to arrays, and hybridized for $17 \mathrm{~h}$ at $42^{\circ} \mathrm{C}$ in single slide hybridization chambers placed in a water bath. A series of $30 \mathrm{~s}$ shaking washes in $2 \times$ SSC plus $0.1 \%$ SDS; $2 \times$ SSC; $0.1 \times$ SSC and Milli$\mathrm{Q}$ water, at room temperature, removed probe excess. The hybridized slides were spin dried and scanned using an Axon Genepix 4000B scanner (Molecular Devices, Sunnyvale, CA) with GENEPIX software (Agilent Technologies, Santa Clara, CA), 10-micron resolution, Cy3 with Green Laser (532 nm), and Cy5 with Red Laser $(635 \mathrm{~nm})$. GenePix Pro 6.0 software was used for quantification of the spots and image analysis with default parameters.

The microarray data analyses were performed with Limma package (Biocondutor project [http://www.bio conductor.org/]) together with R [http://www.r-project. org/] [24]. The Normexp method was used for background normalization adding an offset of 50 to the corrected intensities. Normalization within-arrays was done using print-tip loess to adjust imbalances between the green and red dyes due to spot intensity and spatial position on the slide. Average intensities were normalized between-arrays using the Aquantile method. After normalization, the Log2 ratio of gene expression differences between the developmental phases was calculated. Foldchange values and standard errors were estimated by fitting linear regression to the normalized expression data. The empirical Bayes statistics (moderated t-statistics) was used for differential expression analysis [24]. The p-value of the differentially expressed genes was corrected using FDR $(<0.05)$ [25].

The DEGs were annotated according to Gene Ontology (GO) terms for Biological Process (level 3), Molecular Function (level 2) and Cellular Component (level 3) [26] using DAVID (Database for Annotation, Visualization and Integrated Discovery) Gene Functional Classification Tool (version 6.7) [27]. 


\section{RT-qPCR analysis}

The RNA samples were incubated in the presence of 3 units of RNase-free DNase (Promega) for $40 \mathrm{~min}$ at $37^{\circ} \mathrm{C}$ to eliminate contaminant DNA, and for $15 \mathrm{~min}$ at $70^{\circ} \mathrm{C}$ to inactivate the DNase. First-strand cDNA was synthesized by reverse transcription (RT) using $2.5 \mu \mathrm{g}$ of total RNA, SuperScript II reverse transcriptase and the oligo $(\mathrm{dT})_{12-18}$ primer (Invitrogen, Life Technologies). Reactions not including the SuperScript II reverse transcriptase, or cDNA template, were prepared as negative controls and analyzed in parallel. PCR amplification was carried out at a 7500 Real Time PCR System (Applied Biosystems) using $20 \mu \mathrm{L}$ reaction volumes containing $10 \mu \mathrm{L}$ SYBR Green Master Mix 2x (Applied Biosystems), $1 \mu \mathrm{L}$ cDNA, $7.4 \mu \mathrm{L}$ water, and $0.8 \mu \mathrm{L}$ of each gene-specific primer $(10 \mathrm{mM})$ (Additional file 1: Table S1). The PCR conditions were $50^{\circ} \mathrm{C}$ for $2 \mathrm{~min}$ and $95^{\circ} \mathrm{C}$ for $10 \mathrm{~min}$ followed by 40 cycles of $95^{\circ} \mathrm{C}$ for $15 \mathrm{~s}$, and $60^{\circ} \mathrm{C}$ for $1 \mathrm{~min}$.

When possible (because the $\mathrm{CP}$ genes are in general small and some contain only one intron), the primers used for amplification of the target genes were designed to span an intron, thereby serving as control for genomic DNA contamination. The gene encoding a ribosomal protein, Amrp49, which is expressed in similar levels during the pupal and pharate-adult stages of the honeybee, and was validated as being a suitable reference gene [28], was used for normalizing the RT-qPCR data. The primers for the Amrp49 gene were designed to span one intron.

Each PCR run was followed by melt curve analysis. Primer specificity and absence of prime dimers were confirmed by the sharp melting curve of the PCR product and by the presence of a single band on agarose gel electrophoresis. The efficiency (E) of PCR amplification was calculated from the slope of standard curves (serial dilutions of the cDNA) using the equation $\mathrm{E}=10^{(-1 / \text { slope })}$ and set near 2 .

To check reproducibility, each SYBR green assay was done twice (technical replicate) and repeated with 3 independent samples (biological triplicate). The baseline and threshold were correctly set. The data quantification and normalization relied on the calculation of target threshold cycle $(\mathrm{Ct})$ values and reference gene $\mathrm{Ct}$ values in qBase $^{\text {PLUS }}$ version 2 software [29].

\section{cDNA cloning and sequencing}

The cDNAs obtained from two putative CP genes, GB12449 and GB12811, were amplified using specific primers (Additional file 2: Table S2). PCR products were subjected to electrophoresis on agarose gel, purified with Perfectprep Gel Cleanup kit (Eppendorf), and cloned into pGEM-T Easy Vector (Promega). Insert-containing plasmids were sequenced using M13 forward and reverse universal primers. Dideoxy sequencing was performed in an ABI Prism 310 DNA Analyser using BigDye
Terminator v3.0 Cycle Sequencing Ready Reaction (Applied Biosystems). Sequencher ${ }^{\mathrm{Tm}}$ software version 4.7 (Gene Codes Corporation) was used to analyze the sequences, which were then mapped to the annotated honeybee genome using Artemis software (Release 7, The Sanger Institute).

\section{Fluorescent in situ hybridization}

In situ hybridization was carried out to investigate the presence of GB12449 and GB12811 transcripts in the honeybee epidermis. To construct the fluorescent probes, pairs of specific primers were designed to amplify regions of the GB12449 and GB12811 DNA sequences comprising 300 and 379 nucleotides, respectively (Additional file 2: Table S2). Single-stranded antisense and sense probes were synthesized using the FISH Tag RNA Green kit (Invitrogen, Life Technologies, cat. F32952), following manufacturer's instructions.

Thoracic samples were dissected in cold Ringer saline and fixed in $1 \mathrm{~mL}$ heptane, $80 \mu \mathrm{L}$ HEPES buffer $(0.1 \mathrm{M}$ HEPES, pH 6.9, 2 mM MgSO, 1 mM EGTA), $100 \mu \mathrm{L}$ $8 \%$ paraformaldehyde, $20 \mu \mathrm{L}$ dimethyl sulfoxide (DMSO) for $30 \mathrm{~min}$ under shaking. The fixative was discarded, the samples were quickly rinsed in absolute methanol (2 rinses) and in absolute ethanol (2 rinses), and then stored up to 2 weeks in ethanol at $-20^{\circ} \mathrm{C}$ or immediately rehydrated in PBS pH 7.4 containing $0.1 \%$ Tween-20 (PTw). After an additional fixation during $20 \mathrm{~min}$ in a 9:1 v/v mixture of $4 \%$ paraformaldehyde $/ 0.1 \%$ Triton $\mathrm{X}-100$ and DMSO, the samples were washed in PTw. To facilitate permeabilization and mRNA probe penetration, samples were incubated in a freshly prepared $20 \mu \mathrm{g} / \mathrm{mL}$ proteinase $\mathrm{K}$ in PTw for $5 \mathrm{~min}$, followed by washes in a filter-sterilized $10 \mathrm{mg} / \mathrm{mL}$ glycine solution. Samples were then rinsed in PTw and re-fixed, as above. After washes in PTw, samples were equilibrated in hybridization solution (HS) (50\% formamide, 4x standard saline citrate, 1x Denhardt's solution, $250 \mu \mathrm{g} / \mathrm{mL}$ yeast total RNA, $250 \mu \mathrm{g} / \mathrm{mL}$ boiled salmon testes DNA, $50 \mu \mathrm{g} / \mathrm{mL}$ heparin, 0.1\% Tween 20,5\% dextran sulfate), washed in 1:1 PTw/HS and subsequently in HS. Equilibrated samples were transferred to fresh tubes and pre-hybridized in HS for $1 \mathrm{~h}$ at $45^{\circ} \mathrm{C}$. Sense and antisense probes were separately diluted in $\mathrm{HS}(200 \mathrm{ng} / \mathrm{mL})$, heat-denatured for $2 \mathrm{~min}$ at $98^{\circ} \mathrm{C}$, chilled on ice and added to the pre-hybridized samples. Hybridization was carried-out overnight at $45^{\circ} \mathrm{C}$ under gentle shaking. The hybridized samples were washed in HS: PTw (3:1, 1:1 and 1:3 v/v) and in PTw solution. For cell nuclei co-localization the thoracic samples were stained with diamidino-2-phenylindole (DAPI) diluted 1:4000 in PTw, followed by washes in PTw. The thoracic pieces were transferred to $70 \%$ glycerol in PTw, and mounted on slides using SlowFade ${ }^{\circledR}$ Gold (Invitrogen) for observation under a confocal microscope TCS-SP5 AOBS (Leica). 


\section{Characterization of potential regulatory sequences}

We retrieved functional information from the Gene Ontology (GO) database [26] for the D. melanogaster ortholog genes differentially expressed in the honeybee thoracic dorsum during the pupal-to-adult molt. A pipeline for cis-regulatory motifs discovery was designed based on reliable strategies previously proposed by MacIsaac and Fraenkel [30], and adapted to analyze the honeybee genome $[31,32]$. This pipeline integrates three motif-detection programs: AlignAce [33], MEME [34] and MDscan [35]. Honeybee intergenic databases were constructed for $3 \mathrm{~kb}$ sequence sizes that were trimmed whenever another open reading frame (ORF) was found to be flanking these regions. These databases were exploited for score calculations using group specificity scores (Church scores) [36], ROC-AUC scores [37] and Enrichment scores [38]. Two additional specific score metrics, the MAP score from AlignAce and MDscan and the E-value from MEME, were also used as a first filter for selecting the most significant motifs (MAP $>5$ and E-value $\leq 1 \mathrm{e}-05$ ). The second filter was set up to decrease the amount of spurious hits among the identified DNA motifs (Church $\leq 1 \mathrm{e}-04$, ROC-AUC $\geq 0.7$ and P-value for enrichment $\leq 0.01$ ). The main criterion for identifying known cis-regulatory sites among the overrepresented motifs was the alignment of the PSSM (Position-Specific Scoring Matrix) with the transcription factor binding sites as described in the TRANSFAC database, version 2010.1 [38]. Only the alignments passing a threshold of $70 \%$ identity for each PSSM were considered as significant matches. We used concepts of graph theory and complex networks [39].

We also used the consensus sequence for Fushi Tarazu Transcription Factor 1 (FTZ-F1) binding site (YCAAGG TCR; [40]) to build a PSSM and then identify putative FTZ-F1 binding sites (at least 70\% similar to the consensus sequence) in the $3 \mathrm{~kb}$ upstream intergenic regions of selected DEGs. We used the same score metrics (Church score, ROC-AUC and Enrichment score) to estimate the specificity of FTZ-F1 binding sites.

\section{Results}

\section{Ecdysteroid-coordinated development of the thoracic} dorsum during the pupal-to-adult molt

Figure 1 shows the ecdysteroid titer-coordinated changes in external morphology (exoskeleton) during pupal-toadult transition. Toluidine-stained cross sections of the thoracic dorsal integument and subjacent tissues are also shown for pupae-in-apolysis and two successive pharateadult phases. The ecdysteroid peak induces the detachment of the pupal cuticle from the epidermis. The exuvial fluid is then secreted by the epidermis in the space created beneath the detached pupal cuticle. Next, the adult cuticle (shown in pharate-adults cross sections) is gradually deposited on the epidermis. The thoracic sections of the newly-ecdysed-pupae (not shown) and pupaein-apolysis are histologically very similar, except that in the former the cuticle is still attached to the epidermis, while in the latter it is already detached.

\section{Changes in gene expression in the thoracic dorsum during pupal-to-adult development}

The cDNA microarray analysis revealed 995 and 1,497 spots corresponding to up- or downregulated genes in the thoracic dorsum of pharate-adults compared with newlyecdysed-pupae and pupae-in-apolysis, respectively. These genes represent $6.9 \%$ and $10.4 \%$, respectively, of the 14,400 oligonucleotides spotted on the microarray slides. The oligonucleotide set included the following: Official Gene Set sequences (OGS); variable exons from the antimicrobial peptide apidaecin and from the IG-family gene Dscam; representative genes from viral, fungal, bacterial, and microsporidian pathogens of honeybees; and nonOGS expressed sequence tags (ESTs) from a subtractive library biased toward larval genes upregulated upon exposure to Paenibacillus larvae. Our analyses focused exclusively on the OGS sequences, which included 862 (86.63\%) and 1,304 (87.11\%) genes showing changes in their expression levels in pharate-adults compared with newly-ecdysed-pupae and pupae-in-apolysis, respectively. Some of these oligonucleotides were duplicated in the microarray slides; thus, there were 761 and 1,173 unique DEGs in the respective comparisons. Notably, statistically significant changes in gene expression were not detected during the newly-ecdysed-pupae to pupae-in-apolysis transition; the changes were greater, and therefore statistically significant, after apolysis.

The numbers of DEGs in the thoracic dorsum are shown in Figure 2. Some of these genes were differentially expressed in both comparisons; therefore, we compiled a total of 1,253 unique DEGs. These data highlighted the changes in gene expression during the transition from pupal-to-adult thoracic dorsum. The up- and downregulated genes are listed in Additional file 3: Table S3 and Additional file 4: Table S4.

Figure 3 illustrates the magnitude of the differences in gene expression between the pupal and pharate-adult thoracic dorsum.

\section{Gene ontology (GO) functional analysis}

DAVID was used for GO analysis of DEGs that were orthologous to D. melanogaster genes. The comparisons of pharate-adults with newly-ecdysed-pupae or pupaein-apolysis revealed 705 (92.64\%) and 1,086 (92.58\%) DEGs, respectively, with predicted orthology to $D$. melanogaster genes.

The GO results for the enriched Biological Process, Molecular Function, and Cellular Component categories are shown in Figure 4. DEGs from newly-ecdysed-pupae 


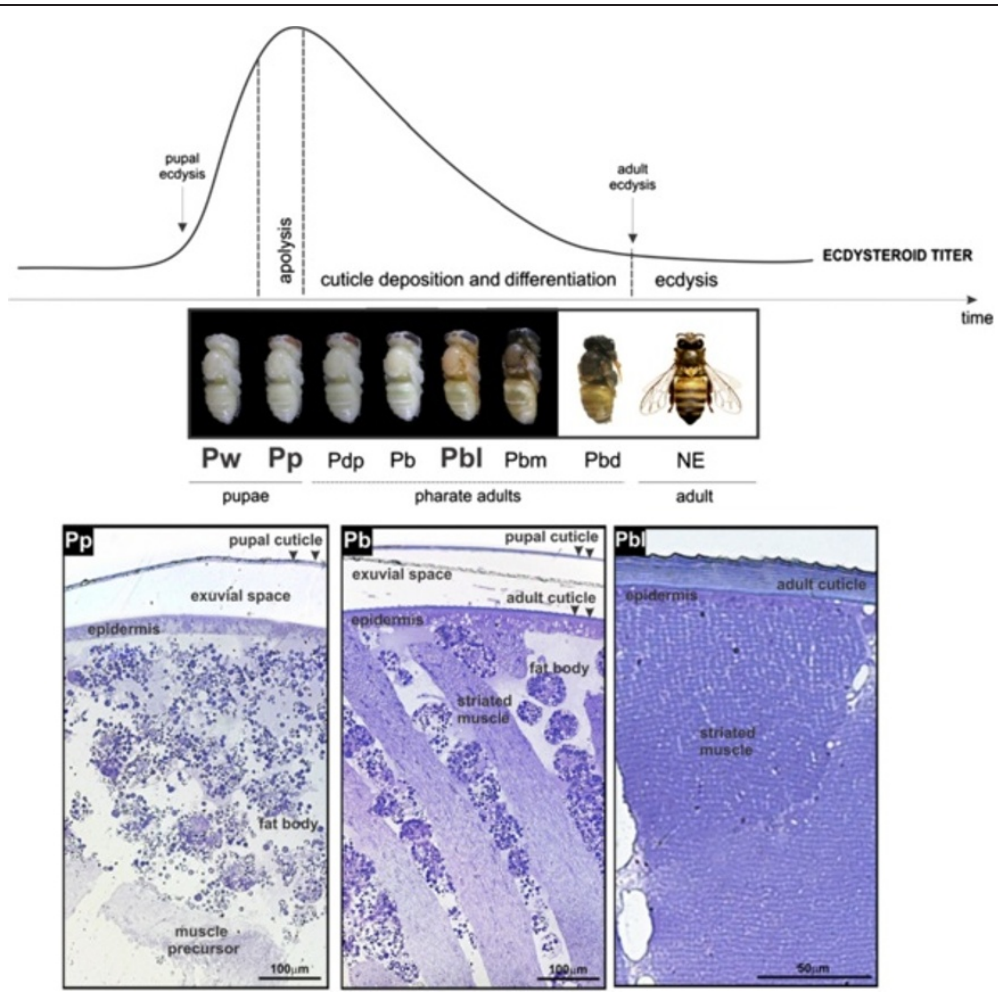

Figure 1 Morphological changes in thoracic dorsum during molting. Thoracic dorsum development in the honeybee during the ecdysteroid-regulated pupal-to-adult molt. Ecdysteroid titer modulation adapted from Pinto et al. [41]. Photos of bees in the sequential phases of the pupal-to-adult molt are shown. The abbreviations in bold under the photos represent the developmental phases used for gene expression analysis: Pw (newly-ecdysed-pupa), Pp (pupa-in-apolysis) and Pbl (pharate-adult). Apolysis is triggered in the Pp phase by the ecdysteroid peak. Deposition and differentiation of the adult exoskeleton occurs under declining ecdysteroid titer in the successive pharate-adult phases (Pdp, Pb, Pbl, Pbm and Pbd). NE: newly-emerged worker bee. Toluidine blue-stained semithin sections of the thoracic dorsum from pupae-in-apolysis (Pp phase), and pharate-adults ( $\mathrm{Pb}$ and Pbl phases) are shown at the bottom. The detached pupal cuticle (arrowheads) is evident in pupae-in-apolysis (Pp phase) and pharate-adults ( $\mathrm{Pb}$ phase). Muscle precursors and abundant fat body cells could be observed in pupae-in-apolysis (Pp phase). Adult cuticle deposition on epidermis (arrowheads), and differentiating muscle fibers interspersed with fat body cells were seen in early pharate-adults (Pb phase). The fat body cells disappear from the thoracic dorsum and muscle fibers contact epidermis in the next pharate-adult phase (Pbl).

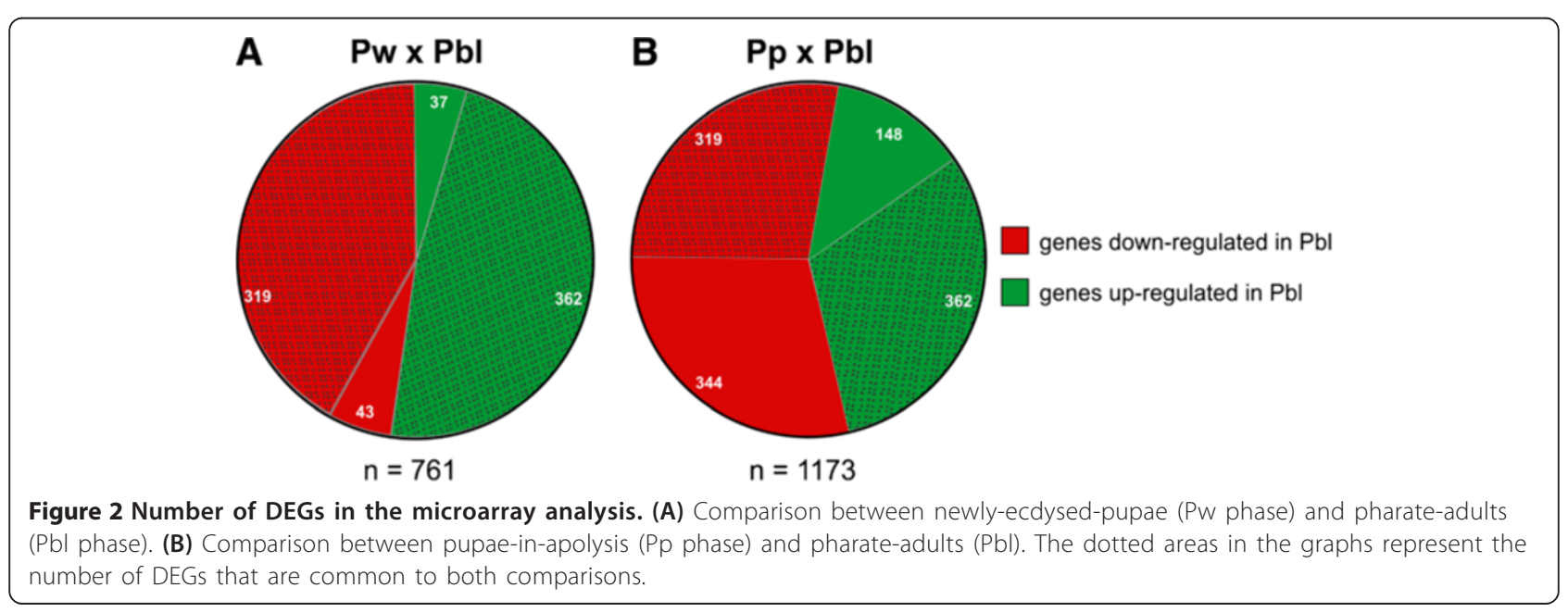



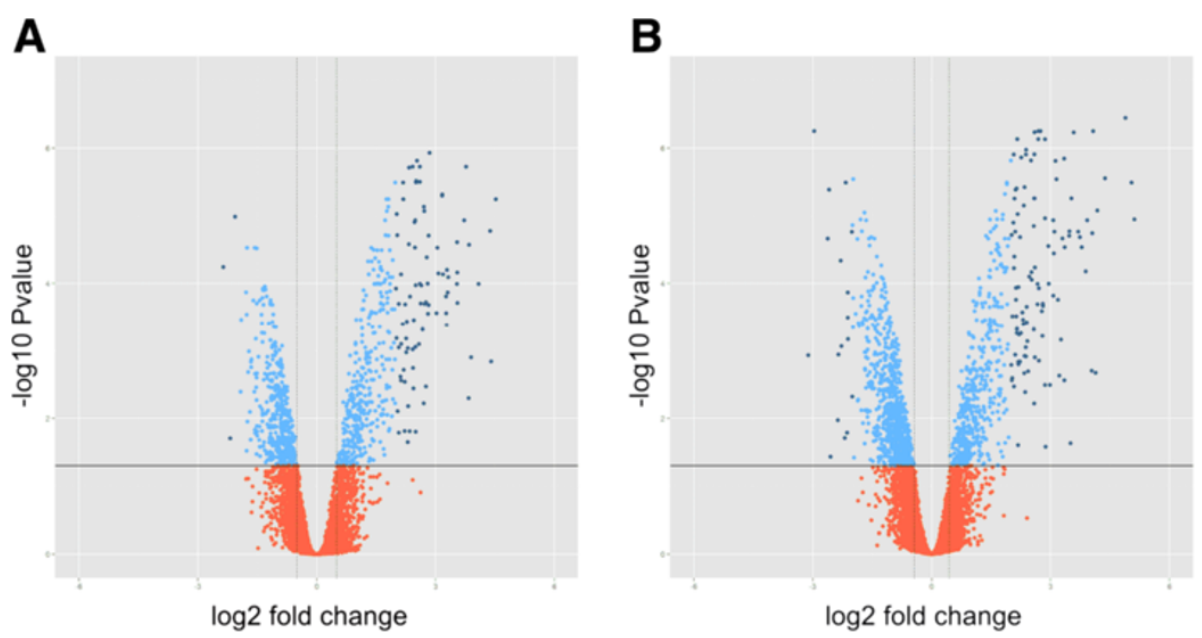

Figure 3 Differential gene expression in the thoracic dorsum during the pupal-to-adult molt. (A) Comparison between newlyecdysed-pupae (Pw phase) and pharate-adults (Pbl phase). (B) Comparison between pupae-in-apolysis (Pp phase) and pharate-adults (Pbl phase). Plots of significance and fold-change are indicated in the $y$ - and $x$-axes, respectively. The horizontal line indicate $p$ value $=0.05$. The genes that display statistically significant differential expression ( $p$ value $<0.05$ ) and high magnitude fold change $($ Log $2 \mathrm{FC}$ cutoff $=0.5)$ are represented as light- and dark-blue dots above the horizontal line (dark-blue dots display Log2 fold-change greater than 2). Red dots below the horizontal line indicate $p$ value $>0.05$ and small magnitude fold change.

and pupae-in-apolysis were grouped in the same Biological Process categories (cellular macromolecule metabolic process; cellular metabolic process involving nucleobases, nucleosides, nucleotides and nucleic acids; macromolecule biosynthetic process; and gene expression including the production of transcripts, translation into proteins, and protein processing events) and Molecular Function (protein binding, nucleic acid binding, and nucleotide binding). By contrast, pharate-adult DEGs were grouped in distinct Biological Process categories (phosphorus metabolic process, electron transport chain, and generation of precursor metabolites and energy), and Molecular Function (structural constituent of cuticle, oxidoreductase activity, substrate specific transporter activity, transmembrane transporter activity, and structural constituent of ribosome) (Figure 4A,B).

Several of the Cellular Component categories were shared by pupal and pharate-adult DEGS, although some of them (membrane, membrane part, organelle membrane, and organelle envelope) were exclusively assigned to pharate-adult DEGs (Figure 4C).

Therefore, GO analysis clearly separated most of the pupal and pharate-adult DEGs into distinct functional categories.

\section{Differentially expressed CP genes}

Within the DEGs, we found 24 genes encoding CPs. Figure 5 shows the classes of differentially expressed CP genes detected in the microarrays. The CPR genes (AmelCPR 3, 4, 6, 14, 15, 17, 23, 24, 25, 28, 29 and 33), which encode proteins containing the chitin-binding $R \& R$
Consensus [13], were the most abundant among the differentially expressed CP genes (50\%). The genes encoding Apidermins (Apd-1, Apd-2, and Apd-3), CPF and CPLCP1 proteins, the Analogous-to-Peritrophins proteins Am-C and Am-D, and the Tweedle1 and Tweedle2 proteins accounted for the remaining differentially expressed CP genes. Another three sequences, GB12449, GB12811 and GB11550, were included in this list of CPs (further details are in the next section).

The structures of the differentially expressed CP genes were determined in silico by mapping them to the annotated honeybee genome using Artemis software (Release 7, The Sanger Institute [42]) (Additional file 5: Figure S1). The differentially expressed $\mathrm{CP}$ genes comprised 2 exons (8 genes), 3 exons (12 genes), or 4-5 exons (4 genes). The first exon is relatively small in 14 of the differentially expressed CP genes. Schematic representations of the clustered CP genes are shown in Additional file 5: Figure S1, and the characteristics of the proteins encoded by the differentially expressed CP genes are described in Additional file 6: Table S5. All 24 CPs contain an N-terminal signal peptide, consistent with their secretion by the epidermis to integrate the cuticle. A low molecular mass was a common feature shared with CPs from other insects. Of the 24 mature honeybee CPs, 21 (87.5\%) have a molecular mass of less than $30 \mathrm{kDa}$. The smallest $\mathrm{CP}$ is $6.1 \mathrm{kDa}$, and the largest is $40.4 \mathrm{kDa}$. The R\&R Consensus-type was identified using hmmfind software, available at the cuticleDB web site [http://bioinformatics2.biol.uoa.gr/cuticleDB/index.jsp], using default parameters. Among the 12 CPR genes identified in the arrays, six contain the RR-1 Consensus sequence, 


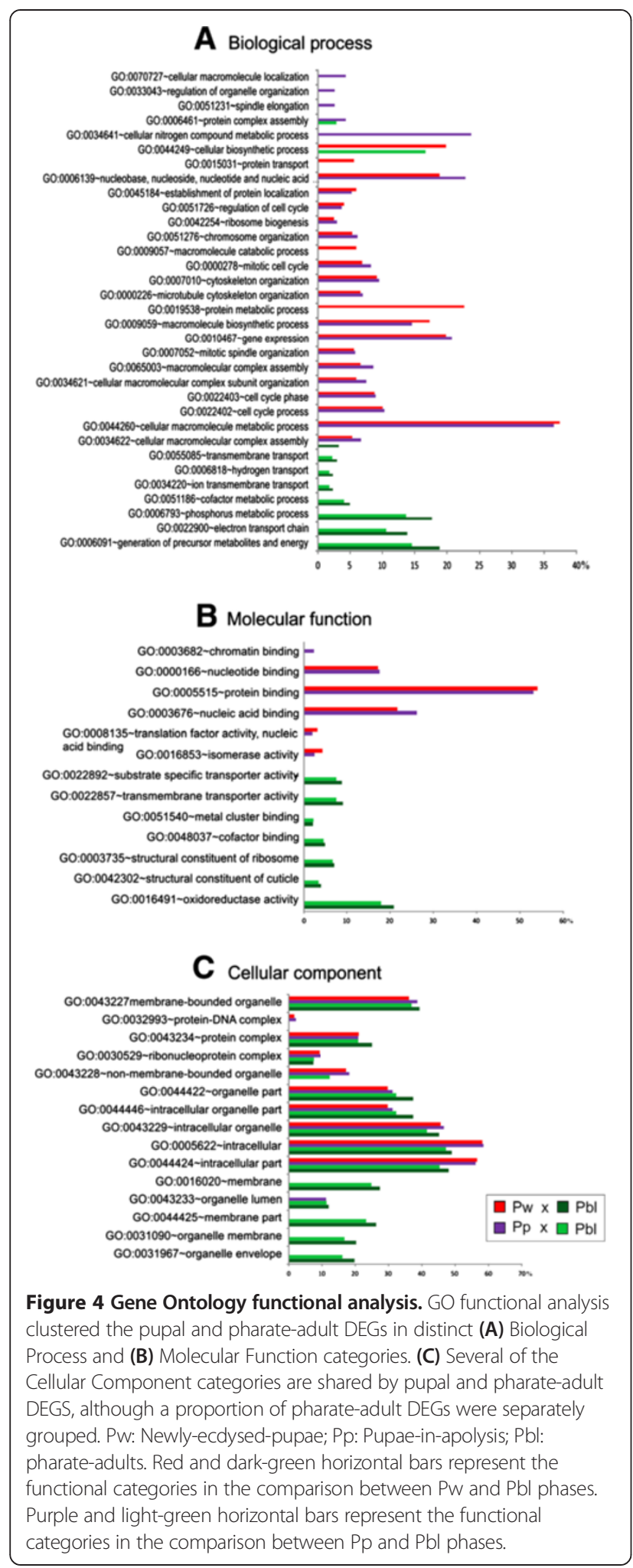

and five have the RR-2 Consensus sequence. We could not determine whether the CPR gene AmelCPR33 has the RR-1, RR-2 or RR-3 Consensus sequence.
The expression (transcript levels) of each of the 24 CP genes was quantified by RT-qPCR using specific primers and thoracic dorsum samples from newly-ecdysed-pupae, pupae-in-apolysis, and pharate-adults. The position of the primers in each predicted gene sequence is illustrated in Additional file 5: Figure S1. Seventy-two comparisons were performed in triplicate with independent samples. The RT-qPCR results agreed with the microarray data in 58 of these comparisons, indicating an accuracy of $80.5 \%$. Except for the gene AmelCPF1 (the unique member of the CPF family revealed in the microarray analysis), all the other $23 \mathrm{CP}$ genes were upregulated in pharate-adults, when the adult cuticle is being deposited (Figure 6).

\section{GB12449 and GB12811 genes are expressed in the epidermis}

The protein FASTA sequence of each DEG was obtained from Amel_prerelease2_OGS_pep data bank (honeybee genome, version 4.0) using GBs as accession numbers. These sequences were analyzed by BLASTP using the non-redundant database (NCBI). The first 10 BLAST hits were searched for the terms "cuticle", "exoskeleton", and "cuticular", which could indicate similarity with described CPs. The predicted proteins encoded by GB12449, GB12811 and GB11550 could not be unequivocally included in any of the 12 previously-defined CP classes [8].

Sequencing of the corresponding cDNAs validated the sequences of GB12449 and GB12811 (Figure 7). Both have features that may qualify them as encoding CPs: (1) abundance of glycine residues (GB12449 and GB12811 sequences have $20.9 \%$ and $22.5 \%$ glycine, respectively); (2) presence of GGYGG and/or GGY motifs, typical of glycine-rich CPs; (3) presence of an N-terminal signal peptide; (4) absence of cysteine residues in the mature protein (GB12811 has a cysteine residue in the signal peptide).

Using in situ hybridization, GB12449 and GB12811 transcripts were localized in the cytoplasm of epidermal cells in the thoracic dorsum of pharate-adults (Figure 8), where these genes were expressed at high levels (see transcript levels in Figure 6). In summary, these genes are expressed in the epidermis and possibly encode proteins involved in cuticle formation.

The protein encoded by GB11550 was identified in integument extracts from honeybee pupae and pharate-adults using 2-dimensional electrophoresis (data not shown). Well-focused protein spots were trypsin-digested, and identified by peptide mass fingerprint using MALDI-TOF mass spectrometry. Mass spectra were analyzed against the NCBI non-redundant protein database using Mascot software for protein identification and characterization. This analysis confirmed the presence of the GB11550 protein in the integument [43]. 


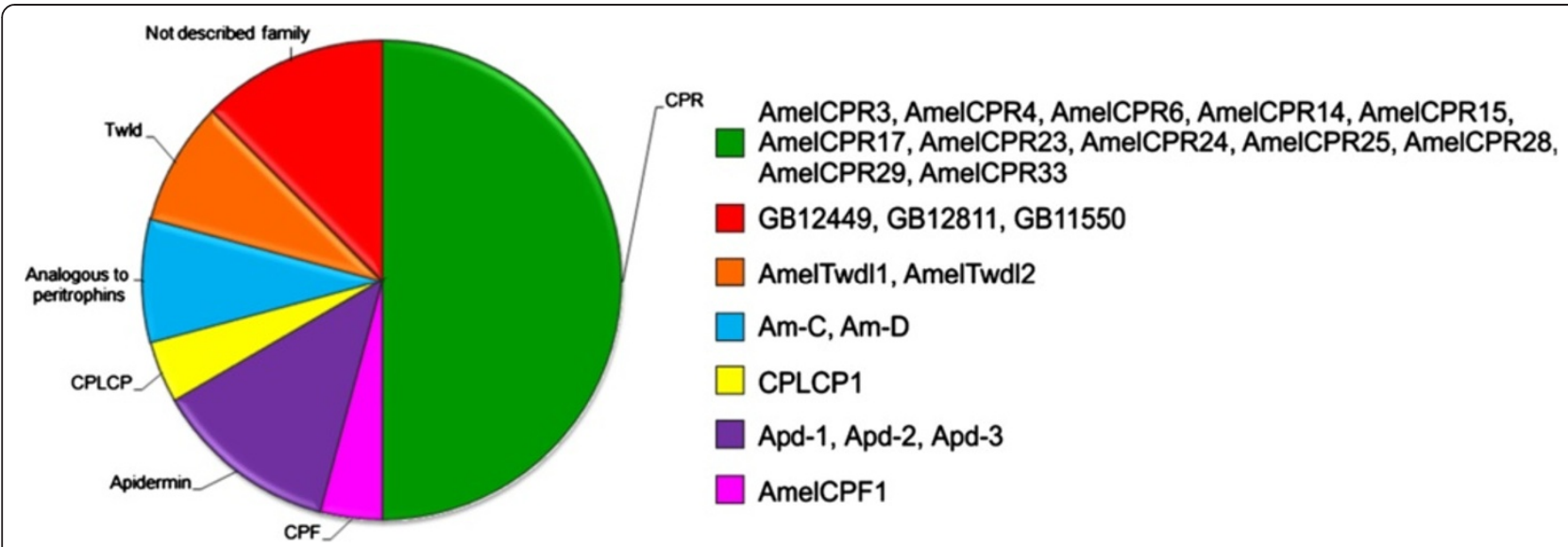

Figure $5 \mathrm{CP}$ genes differentially expressed in the thoracic dorsum. Families of CP genes differentially expressed in the thoracic dorsum. The microarray analysis revealed genes encoding members of CPR, CPF, Apidermin, CPLCP, Analogous-to-Peritrophins, and Tweedle (Twdl) families, in addition to putative CP genes not assigned to any family (GB12449, GB12811, GB11550). Genes in each family are listed at the right.

\section{Differential expression of genes related to thoracic muscle formation}

Among the DEGs, we detected genes that are potentially involved in the differentiation of thoracic muscles from precursor cells. Myoblasts derived from mesenquimal cells are the precursors of myocytes that elongate and fuse to form the adult thoracic muscles [1]. Conventional light microscopy analysis of the thoracic dorsum of pupae-in-apolysis (Figure 1) showed a mass of myocytes subjacent to the integument and surrounded by fat cells. The myocytes differentiate into striated muscle fibers that contact specific regions of the epidermis. Functioning as tendons, the integument infolds (i.e., apodemes) and specific points in the superficial epidermis serve as attachment sites for the striated muscles. Muscle attachment to the epidermis starts in early pharate-adults (at the $\mathrm{Pb}$ phase, which immediately precedes the $\mathrm{Pbl}$ phase). In the thoracic dorsum of the $\mathrm{Pbl}$ phase, the musculature is already in close contact with the integument (Figure 1).

Among the 36 genes identified as "muscle genes" in the DEG list, 28 were upregulated during thoracic musculature formation (Additional file 3: Table S3; Additional file 4: Table S4). These genes are potentially involved in functions such as structural constituents of muscle fibers, muscle tendon junction, muscle attachment, adult somatic muscle development and sarcomere organization.

Before the onset of muscle fibers formation from precursors, the thoracic dorsum is filled with fat body cells (Figure 1: see thoracic cross section of Pp pupain-apolysis). As development proceeds, the fat body cells are gradually replaced by the thoracic musculature (Figure 1, see thoracic cross section of $\mathrm{Pb}$ and $\mathrm{Pbl}$ pharateadults). The hexamerin genes highly expressed in the fat body, hex 70a (GB30362), hex 70b (GB10869), hex 70c (GB13613) and hex 110 (GB14361), were revealed in the microarray analysis of pupal thoracic dorsum. Their transcript levels were reduced in the subsequent developmental phases, when the fat body cells become scant or disappear from the thoracic dorsum (see Additional file 3: Table S3; Additional file 4: Table S4).

In summary, the developmental morphology of the thoracic dorsum was in agreement with the expression of fat body and muscle-related genes in the microarray data.

\section{Regulatory motifs shared by the DEGs}

Thirty motifs resembling previously identified transcription factor binding sites (with greater than $70 \%$ similarity) were discovered in the UCRs of the honeybee DEGs (Additional file 7: Table S6). Ten of these cis-regulatory motifs were shared by several of the upregulated CPand muscle-related genes. The regulatory network shown in Figure 9 organizes all this information and suggested that $\mathrm{CP}$ - and muscle-related genes are co-regulated in the developing thoracic dorsum.

Motif_7 and motif_26 showed greater than 80\% similarity with binding sites for known transcription factors. Motif_7 is $83 \%$ similar to the Serpent (SRP) binding site. SRP is a GATA-like transcription factor involved in the specification of mesoderm-derived tissues in D. melanogaster, including the thoracic musculature. Motif_26 showed 87\% similarity to the Antennapedia (Antp) binding site motif, 85\% similarity to the Engrailed (En) and Zeste (Z) binding site motifs, $82 \%$ similarity to the Giant (Gt) binding site and $88 \%$ similarity to the T-cell-factor (TCF) binding site. Importantly, Antp, En, Z, and TCF are all expressed in the precursors of the thoracic dorsum, i.e., the wing imaginal discs (references in Discussion, part 2).

We also found binding sites sharing more than $70 \%$ similarity with Broad-Complex (BR-C Z1, BR-C Z2 and BR-C Z3 isoforms) response elements (Additional file 7: 


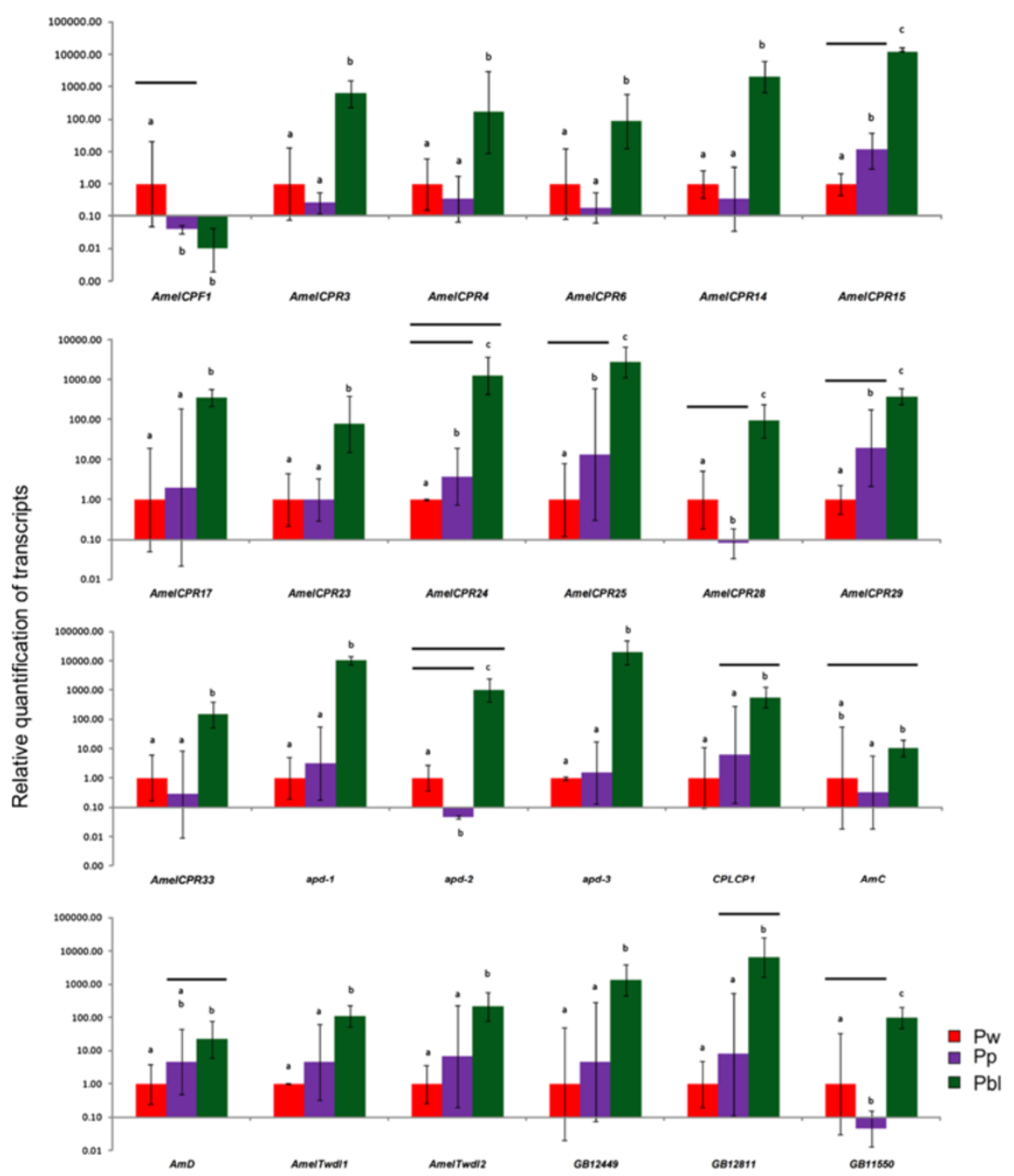

Figure 6 RT-qPCR for quantification of CP transcripts. Relative quantification (RT-qPCR) of transcripts encoding CPs in the thoracic dorsum of newly-ecdysed pupae (Pw), pupae-in-apolysis (Pp), and pharate-adults (Pbl). Overall, the RT-qPCR data are in agreement with the microarray data, except for the comparisons marked by horizontal lines above the graph columns.

Table S6). Broad-Complex isoforms are ecdysone signalrelated transcription factors reported to be important during adult cuticle differentiation [44]. The presence of a BR-C Z3-like binding site (motif 13) in the regulatory region of genes encoding a CP (Tweedle1) and a muscle-related protein (Wnt2) (Figure 9) suggests direct co-regulation by ecdysone during thoracic dorsum formation.

There is experimental evidence that the FTZ-F1 transcription factor regulates the transcription of $\mathrm{CP}$ genes in B. mori and D. melanogaster (reviewed in [45]); therefore, we also searched for FTZ-F1 binding sites in the promoter region of the upregulated $\mathrm{CP}$ - and musclerelated gene set shown in Figure 9. Except for the occurrence of one FTZ-F1-like binding site in the Am-C gene
(GB13298; Figure 9), which is very similar (over 80\%) to the consensus sequence, the presence of FTZ-F1-like binding sites was not supported by any of three enrichment scores used in our computational analysis (pipeline designed for cis-regulatory motifs discovery; Church $>0.05$, ROC-AUC $<0.5$ and Enrichment score $>0.05$ ).

\section{Discussion}

Differential expression of genes involved in thoracic dorsum formation during the ecdysteroid-regulated pupal-to-adult molt

Our microarray data underscored the intense changes in gene expression in the thoracic dorsum during the pupal-to-adult molt, and revealed a set of genes involved in adult cuticle formation. GO analysis clearly grouped 


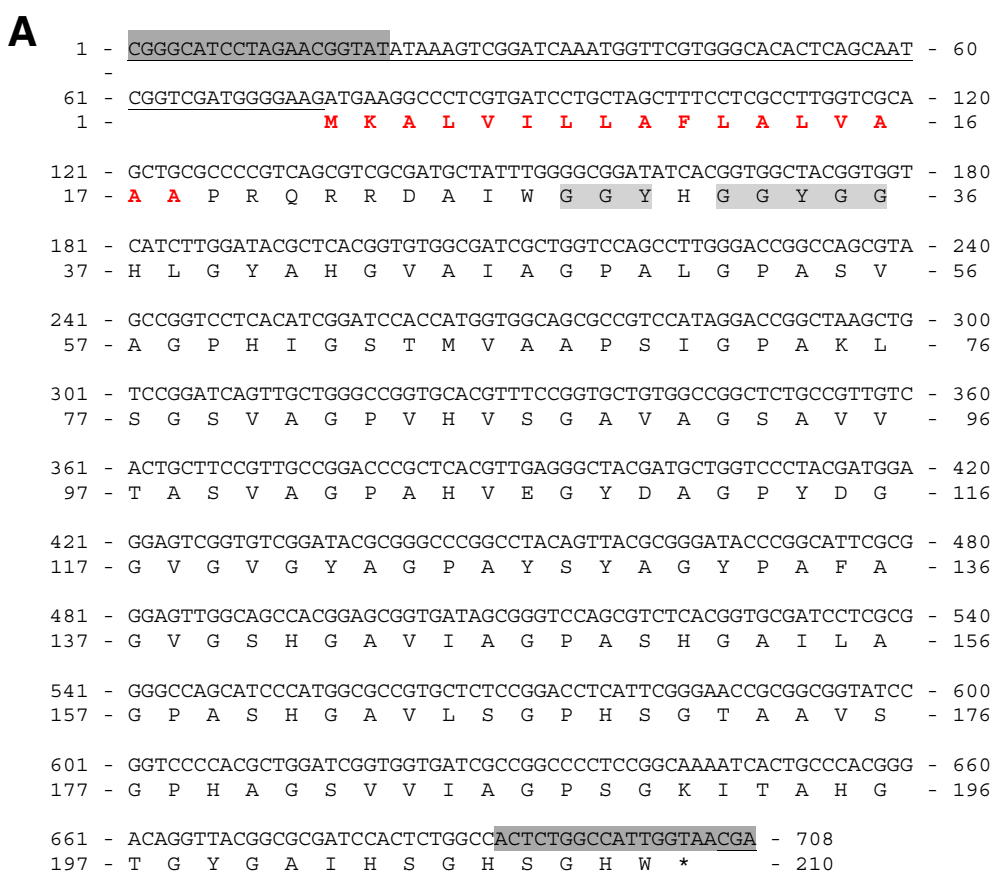

B

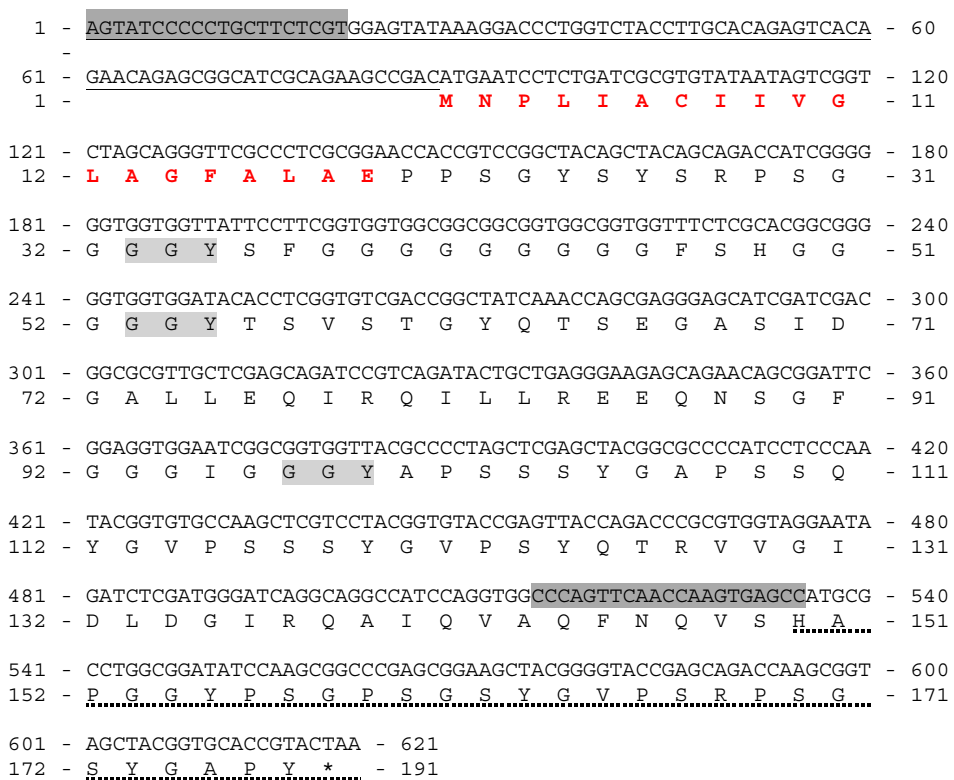

Figure 7 GB12449 (JX456099) and GB12811 (JX456100) cDNA sequences. The genes (A) GB12449 [GenBank:JX456099] and (B) GB12811 [GenBank:JX456100] were validated by sequencing their respective cDNAs. The nucleotides and the predicted amino acid sequences are shown. The $5^{\prime} U T R$ regions are underlined. The primers used for sequencing the genes are marked in dark-gray. Signal peptide is in red letters. Conserved motifs are shown in light-gray. Asterisk indicates stop codon. The non-sequenced portion of GB12811cDNA is marked by a dashed line.

the pupal and pharate-adult DEGs into distinct Biological Process and Molecular Function categories, and revealed the complexity of functions needed for the relatively rapid process of definitive exoskeleton formation.

The most represented GO categories among the pupal DEGs were related to DNA replication, transcription, mRNA splicing and polyadenylation, tRNA metabolic process, rRNA processing, translation, protein folding and Golgi organization. This is in agreement with DNA, RNA and protein syntheses occurring in the epidermis for cell division or increase in size [46]. Other GO categories associated with pupal DEGs (mitotic spindle organization and elongation, cyclin catabolic process, mitotic anaphase-promoting complex activity, regulation 


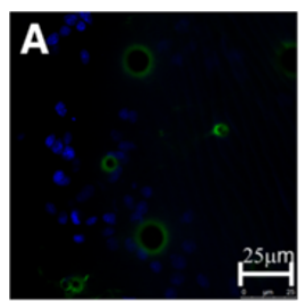

Control

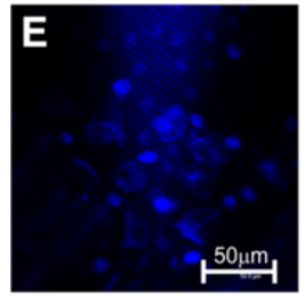

Control

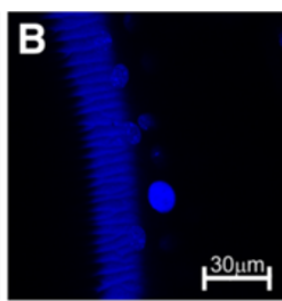

DAPI

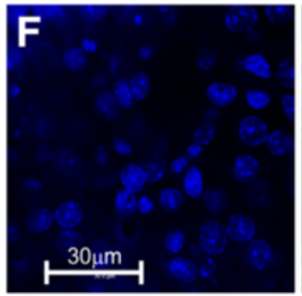

DAPI

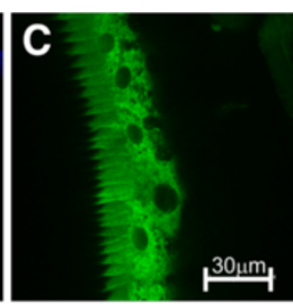

GB12449 FISH

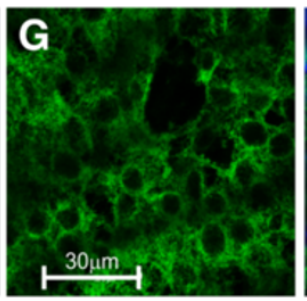

GB12811 FISH

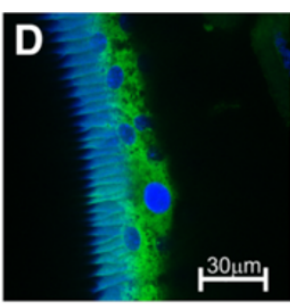

GB12449 FISH + DAPI

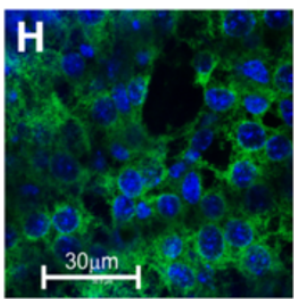

GB12811 FISH + DAPI

Figure 8 FISH for localization of putative CP transcripts in epidermis. Fluorescent in situ hybridization and confocal microscopy showing the presence of GB12449 [GenBank: JX456099] and GB12811 [GenBank:JX456100] transcripts (green) in the epidermis of pharate-adult bees. (A, E) Controls using the sense probes labeled with Alexa Fluor $555^{\circ}$ (Invitrogen) and DAPI to stain cell nuclei; (B, F) DAPI-stained cell nuclei (blue); (C, G) GB12449 and GB12811 antisense probes labeled with Alexa Fluor 555 (Invitrogen); (D, H) Merged B+ C and F+ G images. A, $\mathbf{E}-\mathbf{H}$ : monolayer of epidermal cells in focal planes. B-D: cross sections of the integument showing the cuticle at the left and the epidermis at the right. Setal sockets (green rings in $\mathbf{A}$ ) and the cuticle (seen in $\mathbf{B}-\mathbf{D}$ ) are self-fluorescent.

of mitotic metaphase/anaphase transition, transcription involved in G2/M phase of mitotic cell cycle and regulation of cell cycle) suggest roles in cell division. Such activities precede, and are usually correlated with, the increase in ecdysteroid titer and apolysis. Furthermore, secretion and membrane trafficking apparently occurs in response to ecdysone, which has been reported to induce Golgi formation (reviewed in [47]).

By contrast, the most represented GO categories among pharate-adult DEGs were mainly related to mitochondrial activity and energy generation (mitochondrial electron transport, oxidative phosphorylation, and ATP synthesis coupled electron transport). The GO categories including structural constituent of the cuticle, peroxidase activity, oxidoreductase activity and transmembrane and protein transport, could be intuitively connected to adult cuticle deposition and differentiation.

The most prominent epidermal products involved in cuticle formation are the structural CPs. Based on sequence motifs, the CPs identified to date in insects and other arthropods were tentatively classified into 12 different families [8]. Only six of the $12 \mathrm{CP}$ families have been predicted in the honeybee genome. Proteins in these six families are encoded by $47 \mathrm{CP}$ genes, of which 32 have the $R \& R$ Consensus sequence characteristic of the CPR family, three encode proteins from the CPF/ CPF-like families [48], two encode Tweedle proteins [49], two encode CPLCP proteins (Cuticular Protein of Low Complexity, Proline-rich [50]), three encode Apidermins
[14] and five encode CPAP3 family proteins (Cuticular Proteins Analogous to Peritrophins having 3 ChtBD2 chitin-binding domains) [51].

The whole-genome oligonucleotide array analysis revealed 24 CP genes, including six that were previously validated $[12,14,15]$. All but one (AmelCPF1) of these genes was upregulated during the synthesis and deposition of the adult cuticle. Twelve of them encode CPR proteins. Eight genes encode CPs from the Apidermin, CPLCP, Analogous-to-Peritrophin and Tweedle families. The other three upregulated genes (GB12449, GB12811 and GB11550) could not be categorized into a CP family. However, GB12449 and GB12811 sequences showed motifs and features that have been frequently used to identify putative CP genes in insect genomes. Furthermore, in situ hybridization experiments showed that GB12449 and GB12811 transcripts are abundant in the epidermis during adult cuticle deposition, suggesting that their products are structural CPs. The GB11550 translation product was identified in the integument by 2-dimensional electrophoresis and mass spectrometry, and was found in higher levels in pharate-adults than in pupae [43]. The upregulation of these $23 \mathrm{CP}$ genes in pharate-adults, as verified in the microarray data, was confirmed by RT-qPCR transcript quantification. All these genes are induced after apolysis, i.e., after the ecdysteroid pulse, coinciding with adult cuticle deposition.

As revealed by microarray hybridization and RT-qPCR analyses, AmelCPF1 did not fit the typical pattern of 


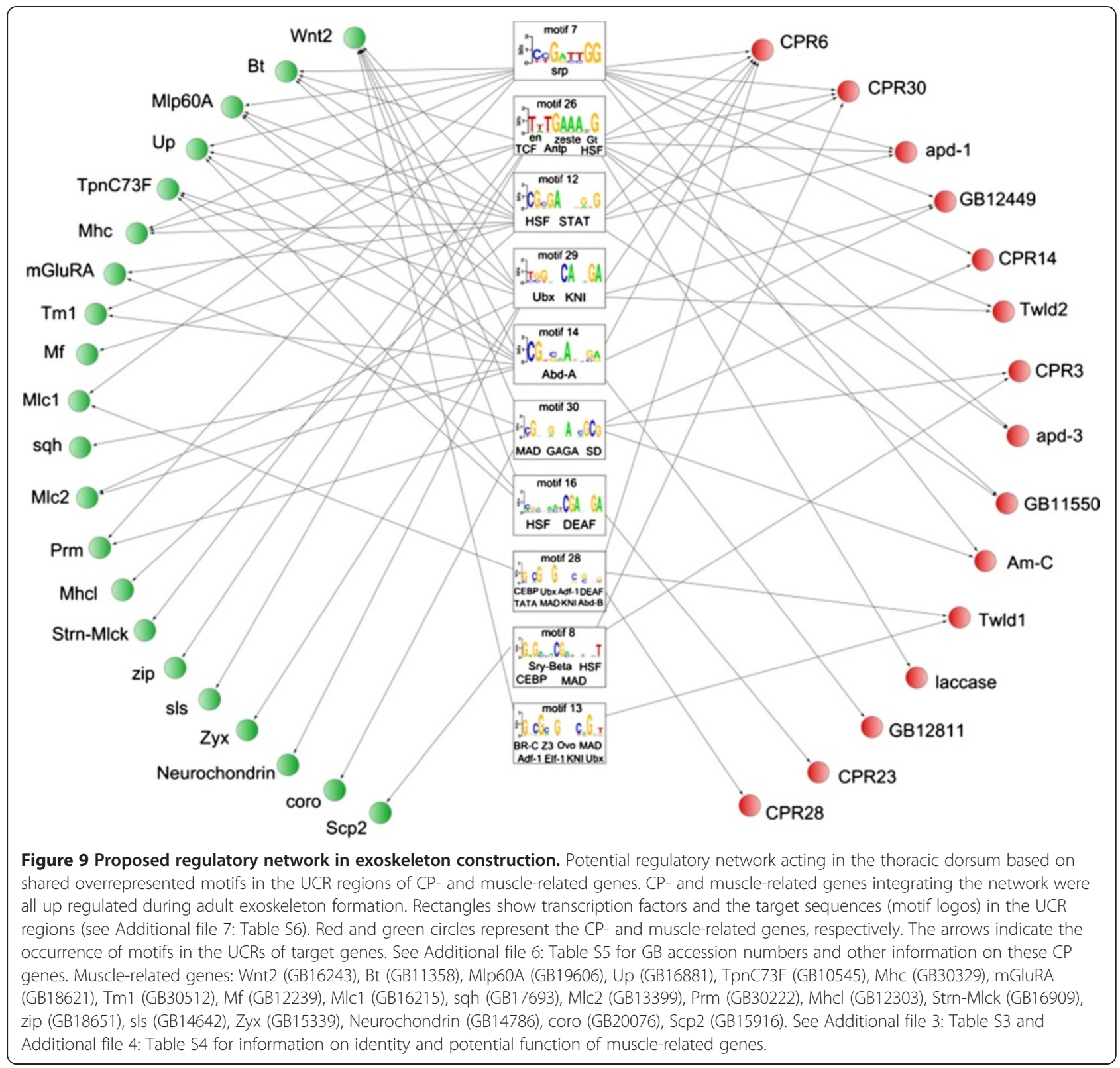

$\mathrm{CP}$ gene regulation because its expression is higher in the integument of newly-ecdysed-pupae than in pupae-in-apolysis and pharate-adults. This suggests that AmelCPF1 is downregulated during apolysis by the ecdysteroid peak. This gene is apparently not involved in adult thoracic cuticle formation, although it may have a role in structuring the pupal cuticle. Using stringent criteria for characterizing stage-specific CPR family genes in Anopheles gambiae, Togawa et al. [10] identified genes that appeared to be specific to a single developmental stage, namely, the larval, pupal, or adult stages. The investigation of AmelCPF1 expression in other developmental stages, or instars, may help to elucidate its stage-specific character.
The RT-qPCR findings highlighted the transient and statistically significant downregulation of AmelCPR28, apd-2 and GB11550 during apolysis (see Figure 6), when the ecdysteroid titer is maximal. Several CP genes are downregulated in vivo by the high ecdysteroid titer that induces apolysis, or in vitro by a high ecdysteroid concentration; expression is then recovered following the hormone peak, or after its removal from the incubation medium (reviewed in [45]). The ecdysteroid peak seems important for reprogramming these genes, and other genes involved in the molting process, for later expression. This type of ecdysteroid-modulated expression was previously demonstrated for AmelCPR14 [12], AmelTwdl1 and AmelTwdl2 [15]. The microarray and RT-qPCR findings 
have extended the number of $\mathrm{CP}$ genes that are potentially upregulated by the ecdysteroid pulse for construction of the adult exoskeleton.

The CPR genes upregulated in the thoracic dorsum of pharate-adults encode proteins containing RR-1 (6 genes) or RR-2 (5 genes) Consensus sequences, implying that both contribute to the structure of the hard (rigid) thoracic cuticle. This finding contrasts with earlier studies connecting RR-1 to soft (flexible) cuticles and RR-2 to hard cuticles. This is a subject not yet fully resolved, because studies on cuticle genes and proteins have been mainly made with whole body extracts. Andersen [52] suggested that RR-2 proteins are part of the exocuticle deposited during the pharate stage, whereas RR-1 proteins integrate the postecdysial endocuticle. However, this hypothesis has not yet been unequivocally validated $[8,10]$.

The differential expression of genes encoding enzymes such as prophenoloxidase, tyrosine hydroxylase, and dopadecarboxylase, which catalyze reactions leading to cuticle melanization and sclerotization, was not detected by the microarray analysis. We previously used RT-qPCR to investigate the expression of these genes in the honeybee integument [16,53]; there were significant increases in transcript levels in pharate-adults ( $\mathrm{Pbm}$ and $\mathrm{Pbd}$ phases) older than those used in the current study (Pbl phase) (see the developmental phases in Figure 1). Consequently, the genes encoding these enzymes were not detected among the DEGs in our microarray analysis. However, other genes potentially involved in cuticle tanning, such as those encoding a laccase (Amlac2) and five peroxidases, were upregulated in pharate-adults. Amlac2 encodes a laccase involved in the cross-linking of CPs and quinones for cuticle sclerotization [54]. Two of the peroxidase genes, GB13459 and GB10387, displayed 20\% and 32\% similarity (ClustalW 2.1 score), respectively, at the protein level with the translation product of a previously described cuticular peroxidase gene, Ampxd [GenBank:ADE45321.2] [15]. These Animal Heme Domain-containing peroxidases catalyze oxidative reactions, and may play a role in the oxidation of catechols to quinones, which leads to cuticle sclerotization $[55,56]$.

The other peroxidase genes included a glutathione peroxidase (GB14138) and two peroxiredoxins (GB10498 and GB10972), also known as thioredoxin peroxidases. The honeybee glutathione peroxidase gene was previously included in a study on the evolution of this gene family in invertebrates [57]. Glutathione peroxidases and peroxiredoxins have been characterized in numerous taxa as important antioxidants. Both proteins were identified in the cuticle of parasitic nematodes, possibly for protection against reactive oxygen species generated by the defense system of the host (reviewed in [58]).

Like the $\mathrm{CP}$ genes and peroxidase genes identified in the microarray analysis, Amlac2 and Ampxd displayed increased expression in pharate-adults, following the ecdysteroid pulse.

\section{$\mathrm{CP}$ - and muscle-related genes in the thoracic dorsum may share common regulatory motifs}

Studies in Drosophila have shown that the notum epidermis and subjacent muscle fibers share the same precursors, i.e., both develop from the wing imaginal discs [23]). The epithelial cells of the discs are ectodermal in origin and differentiate into the notum and wing epidermis. Mesodermal adepithelial cells associated with the wing imaginal discs give rise to myoblast precursors of flight muscles [59]. Ten of the 30 potential binding sites for known transcription factors in the TRANSFAC database were shared by upregulated CP- and muscle-related genes (see Figure 9). Thus, these genes may be co-regulated during the differentiation of the thoracic exoskeleton. Two of these motifs, motif_7 and motif_26 (see Figure 9), are noteworthy because they are more than $80 \%$ similar to binding site motifs for known transcription factors.

Motif 7 is similar to the SRP transcription factor binding site. SRP is important for differentiation of mesodermderived tissues, such as the fat body and muscle precursors, in D. melanogaster embryos [60], and may downregulate the expression of a gene essential for myogenesis: Mef2 (Myocyte-specific enhancer factor 2) [61,62].

Motif_26 is highly similar to binding sites for transcription factors active in developing wing imaginal discs in Drosophila, such as Antp, Z, En, and TCF. Antp is expressed in the presumptive notum (thoracic dorsum) region [63]. During metamorphosis, $z$ is expressed in all imaginal disc derivatives and in the thoracic musculature of pharate-adults [64]. en plays a major role in the territorial subdivision of the imaginal wing discs for wing and thoracic dorsum (notum) definition (reviewed in [65]). dTCF (also called Pangolin) mediates the Wingless (Wg) signal transduction pathway in the wing imaginal discs. Like en, Wg is a player in wing disc territorial subdivision, and is involved in intercellular signaling that specifies the wing and notum primordia $[66,67]$. It is likely that factors active in the precursors (wing imaginal discs) remain active in the tissues derived from them (thoracic dorsum). This is an inference awaiting functional analysis.

Several of the potential transcription factor binding sites identified in the honeybee DEGs were previously found in A. gambiae CPR genes [10], such as Zen, Gt, Tll, Eve, Ubx, Abd-B, Twi, Z, and Broad-Complex isoforms (BR-C Z1, Z2, and Z3), the ecdysone signaling-related transcription factors. There is experimental evidence that Broad-Complex proteins regulate the transcription of $\mathrm{CP}$ genes in B. mori $[68,69]$.

The presence of binding sites for BR-C isoforms suggests that ecdysone directly regulates some DEGs during 
thoracic dorsum formation. As we previously demonstrated, the expression of the CP gene AmelTwdl1 is modulated by the ecdysteroid titer [15]. The discovery of a potential BR-C Z3-like binding site motif in the AmelTwdl UCR (motif_13 in Figure 9) is consistent with this finding. In $D$. melanogaster, the attachment of thoracic muscles to the dorsal epidermis requires $\mathrm{BR}-\mathrm{C} \mathrm{Z1}$ function. It was proposed that $B R-C Z 1$ is induced by ecdysteroids in the thoracic wall and regulates target genes involved in tendon cell maturation [70]. Ecdysteroids also plays a key role in adult muscle development [71]. The muscle-related gene Wnt2 has a potential BR-C Z3-like binding site motif (motif_13 in Figure 9), suggesting its regulation by ecdysteroids. In Drosophila Wnt2 mutants, flight muscles are lost or fail to attach to their epidermal targets during adult thoracic muscle formation [72].

In addition to tweedle 1 and Wnt2, other upregulated $\mathrm{CP}$ - and muscle-related DEGs contain potential binding sites for BR-C isoforms. Motif_25, which is similar to the BR-C Z1 binding site, was identified in the CP gene encoding an apidermin, apd-2; motif_9, a possible bind-

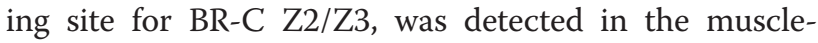
related gene myofilin. Similarly, putative binding sites for BR-C Z2 (motif_15) were identified in genes encoding a peroxidase and a peroxiredoxin. Both enzymes have been associated with cuticle pigmentation and sclerotization $[15,56]$, which are regulated by ecdysteroids [73,74]. These motifs were omitted from the regulatory network (Figure 9) because they were identified in CP- or muscle-related genes, and the regulatory network was constructed exclusively with those motifs detected in both $\mathrm{CP}$ - and musclerelated genes.

Functional analysis has shown that FTZ-F1 regulates the transcription of CP genes in B. mori and D. melanogaster (reviewed in [45]). Although FTZ-F1 binding sites are not enriched in the honeybee $\mathrm{CP}$ genes, the occurrence of a putative site in the Am-C gene (GB13298; Figure 9) may suggest this cuticular gene is regulated by FTZ-F1.

Our bioinformatics approach only hints at the complex regulatory network supposedly involved in thoracic exoskeleton development. By integrating our data in a gene network (Figure 9) we provide a hypothetical framework, which can be the basis for further functional analyses.

\section{Conclusions}

Our data broadens our understanding on thoracic dorsum formation, at the molecular level, during the pupalto-adult molt. Gene expression changes associated with formation of the hard thoracic dorsum in the honeybee were demonstrated using genome-wide cDNA microarray analysis and RT-qPCR. Of the 1,253 DEGs, 547 were upregulated in the thoracic dorsum following apolysis, implying induction by the ecdysteroid pulse that triggers cuticle deposition. CP genes potentially encoding proteins of the CPR, Tweedle, Apidermin, CPF, CPLCP1, and Analogous-to-Peritrophins families were associated with thoracic dorsum formation. Three other genes, which do not belong to any known $\mathrm{CP}$ families, are novel CP candidate genes. In support of this hypothesis, fluorescent in situ hybridization revealed abundant expression of two of these novel genes in the pharate-adult epidermis, which is actively engaged in adult exoskeleton synthesis. Several potential binding sites for biologically relevant transcription factors were shared by upregulated $\mathrm{CP}$ - and muscle-related genes, suggesting co-regulation for coordinated development of thoracic exoskeleton and subjacent striated musculature. Further functional analysis of these cis-elements will be needed to test this hypothesis.

\section{Additional files}

\begin{abstract}
Additional file 1: Table S1. Primers used for the RT-qPCR analysis.
Additional file 2: Table S2. Primers used for Fluorescent in situ hybridizations and sequencing.
\end{abstract}

Additional file 3: Table S3. Genes differentially expressed in the comparison between newly-ecdysed-pupae (Pw phase) and pharateadults (Pbl phase)

Additional file 4: Table S4. Genes differentially expressed in the comparison between pupae-in-apolysis (Pp phase) and pharate-adults (Pbl phase).

Additional file 5: Figure S1. Schematic representation of the structure of the differentially expressed CP genes.

Additional file 6: Table S5. Differentially expressed CP genes and characteristics of their deduced proteins.

Additional file 7: Table S6. Thirty motifs (cis-elements) sharing more than $70 \%$ similarity with binding sites for known transcription factors.

\section{Abbreviations}

Abd-B: Abdominal-B; Antp: Antennapedia; ATP: Adenosine triphosphate; BR-C Z1, BR-C Z2, BR-C Z3: Broad-Complex isoforms; cDNA: Complementary DNA; CP: Cuticular protein; CPAP3: Cuticular protein analogous to peritrophins having 3 ChtBD2 chitin-binding domains; CPF: Cuticular protein containing a 41-44 aminoacids conserved sequence; CPLCP: Cuticular protein of low complexity- proline-rich; CPR: Cuticular protein containing a Rebers and Riddiford Consensus; Ct: Threshold cycle; DAPI: Diamidino-2-phenylindole; DAVID: The data base for annotation, visualization and integrated discovery; DMSO: Dimethyl sulfoxide; DNAse: Deoxyribonuclease; DEG: Differentially expressed gene; DNA: Deoxyribonucleic acid; dTCF: Drosophila T-cell factor; En: Engrailed; Eve: Even-skipped; FDR: False discovery rate; FTZ-F1: Fushitarazu factor 1; GEO: Gene expression omnibus database; GO: Gene ontology; Gt: Giant; HS: Hybridization solution; mRNA: Messenger ribonucleic acid; Mef2: Myocyte-specific enhancer factor 2; MIAME: Minimum information about a microarray experiment; NCBI: National center for biotechnology information; OGS: Official gene set sequences; ORF: Open reading frame; $\mathrm{Pb}$ : Pbl, Pbm, Pbd, Successive pharate adult phases in honeybee development; PBS: Phosphate buffered saline; PCR: Polymerase chain reaction; Pp: Honeybee pupae-in-apolysis; PSSM: Position-specific scoring matrix; Ptw: Phosphate buffered saline containing Tween-20; Pw: Honeybee newly-ecdysed pupae; rRNA: Ribosomal ribonucleic acid; RNA: Ribonucleic acid; RR-1: RR-2, R\&R Consensus types; RT: Reverse transcription; RTqPCR: Real-time reverse transcription polymerase chain reaction; SDS: Sodium dodecyl sulfate; SRP: Serpent transcription factor; SSC: Saline sodium citrate buffer; TCF: T-cell factor; TIl: Tailless; tRNA: Transfer ribonucleic acid; Twi: Twist; Ubx: Ultrabithorax; UCR: Upstream control region; UV: Ultraviolet light; Wg: Wingless; Z: Zeste; Zen: Zerknüllt. 


\section{Competing interests}

The authors declare that they have no competing interests.

\section{Authors' contributions}

MPMS and ARB performed the microarray analysis. ACQS analyzed the microarray data. MPMS performed the RT-qPCR analysis, fluorescent in situ hybridization, and prepared all figures and additional files. ACS and FCPF performed the motif analyses and constructed the regulatory network. LLC provided the integument histological sections. MMGB designed the study and wrote the manuscript. All authors read and approved the final manuscript.

\section{Acknowledgements}

This research was supported by the Fundação de Amparo à Pesquisa do Estado de São Paulo (FAPESP: 10/16380-9; 11/03171-5), which also provided a fellowship (07/04314-9) to M. P. M. Soares. We are very grateful to Dr. A. D. Bomtorin and Dr. R. P. Dallacqua for their help with the microarray and FISH experiments, and Dr. D. G. Pinheiro for bioinformatics support. We thank Prof. Dr. Z. L. P. Simões for valuable comments on project design, and two anonymous reviewers for helpful comments on the manuscript. We also thank J. C. McNamara for reading the Abstract and L. R. Aguiar for his valuable technical assistance in the apiary.

\section{Author details}

'Departamento de Genética, Faculdade de Medicina de Ribeirão Preto, Universidade de São Paulo, Ribeirão Preto, SP, Brasil. ${ }^{2}$ Departamento de Biologia Celular, Tecidual e do Desenvolvimento, Instituto de Ciências Biomédicas, Universidade Federal de Alfenas, Alfenas, MG, Brazil. ${ }^{3}$ Centro de Engenharia, Modelagem e Ciências Sociais Aplicadas - CECS, Universidade Federal do ABC, Santo André, SP, Brazil. "The Queensland Brain Institute, The University of Queensland, Brisbane, Queensland, Australia. ${ }^{5}$ Departamento de Biologia, Faculdade de Filosofia, Ciências e Letras de Ribeirão Preto, Universidade de São Paulo, Ribeirão Preto, SP, Brasil.

Received: 12 March 2013 Accepted: 23 August 2013

Published: 28 August 2013

\section{References}

1. Snodgrass RE: Anatomy of the honey bee. Ithaca: Cornell University; 1956.

2. Thompson PR: Histological development of cuticle in the worker honeybee, Apis mellifera adansonii. J Apic Res 1978, 17:32-40.

3. Michelette ERF, Soares AEE: Characterization of preimaginal developmental stages in Africanized honey bee workers (Apis mellifera L). Apidologie 1993, 24:431-440.

4. Elias-Neto M, Soares MPM, Bitondi MMG: Changes in integument structure during the imaginal molt of the honey bee. Apidologie 2009, 40:29-39.

5. Hepburn HR: Structure of the integument. In Comprehensive Insect Physiology, Biochemistry and Pharmacology. Volume 3. Edited by Kerkut GA, Gilbert LI. Oxford: Pergamon Press; 1985:1-58.

6. Vincent JFV, Wegst UGK: Design and mechanical properties of insect cuticle. Arthropod Struct Dev 2004, 33:187-199.

7. Willis JH, Iconomidou V, Smith RF, Hamodrakas SJ: Cuticular proteins. In Comprehensive Molecular Insect Science. Volume 4. Edited by Gilbert L, latrou K, Gill S. Oxford: Elsevier; 2005:79-110.

8. Willis $\mathrm{JH}$ : Structural cuticular proteins from arthropods: annotation, nomenclature, and sequence characteristics in the genomics era. Insect Biochem Mol Biol 2010, 40:189-204.

9. Togawa T, Augustine Dunn W, Emmons AC, Willis JH: CPF and CPFL, two related gene families encoding cuticular proteins of Anopheles gambiae and other insects. Insect Biochem Mol Biol 2007, 37:675-688.

10. Togawa T, Dunn WA, Emmons AC, Nagao J, Willis JH: Developmental expression patterns of cuticular protein genes with the R\&R Consensus from Anopheles gambiae. Insect Biochem Mol Biol 2008, 38:508-519.

11. Cornman RS, Willis $\mathrm{JH}$ : Annotation and analysis of low-complexity protein families of Anopheles gambiae that are associated with cuticle. Insect $\mathrm{Mol}$ Biol 2009, 18:607-622.

12. Soares MPM, Elias-Neto M, Simões ZL, Bitondi MM: A cuticle protein gene in the honeybee: expression during development and in relation to the ecdysteroid titer. Insect Biochem Mol Biol 2007, 37:1272-1282.

13. Rebers JE, Riddiford LM: Structure and expression of a Manduca sexta larval cuticle gene homologous to Drosophila cuticle genes. J Mol Biol 1988, 203:411-423.
14. Kucharski R, Maleszka J, Maleszka R: Novel cuticular proteins revealed by the honey bee genome. Insect Biochem Mol Biol 2007, 37:128-134.

15. Soares MPM, Silva-Torres FA, Elias-Neto M, Nunes FMF, Simões ZLP, Bitondi MMG: Ecdysteroid-dependent expression of the tweedle and peroxidase genes during adult cuticle formation in the honey bee Apis mellifera. PLoS One 2011, 6:e20513.

16. Canhos LL: Caracterização de mudanças subcelulares e da expressão dos genes codificadores das enzimas tirosina hidroxilase e dopa descarboxilase no tegumento em diferenciação da abelha Apis mellifera. Monografia. Ribeirão Preto, SP, Brazil: Universidade de São Paulo, Departamento de Biologia; 2010.

17. Ote M, Mita K, Kawasaki H, Seki M, Nohata J, Kobayashi M, Shimada T: Microarray analysis of gene expression profiles in wing discs of Bombyx mori during pupal ecdysis. Insect Biochem Mol Biol 2004, 34:775-784.

18. Liang J, Zhang L, Xiang Z, He N: Expression profile of cuticular genes of silkworm Bombyx mori. BMC Genomics 2010, 11:173.

19. Dittmer NT, Hiromasa Y, Tomich JM, Lu N, Beeman RW, Kramer KJ, Kanost MR: Proteomic and transcriptomic analyses of rigid and membranous cuticles and epidermis from the elytra and hindwings of the red flour beetle Tribolium castaneum. J Proteome Res 2012, 11:269-278.

20. Minckley RL: Comparative morphology of the mesosomal 'gland' in male large carpenter bees (Apidae: Xylocopini). Biol J Linn Soc 1994, 53:291-308.

21. Cruz-Landim C, Reginato RD: Preliminar report on the presence of tegumentar glands in the thorax of Meliponinae bees (Hymenoptera, Apidae). Rev Bras Biol 1999, 59:167-172.

22. Vinson SB: Ultrastructure of the mesosomal gland of Xylocopa micans Lepeletier (Hymenoptera: Anthophoridae) associated with pheromone release. Int J Insect Morphol Embryol 1994, 23:243-252.

23. Lawrence PA, Brower DL: Myoblasts from Drosophila wing disks can contribute to developing muscles throughout the fly. Nat 1982, 295:55-57.

24. Smyth GK: Limma: linear models for microarray data. In Bioinformatics and Computational Biology Solutions using $R$ and Bioconductor. Edited by Gentleman R, Vincent JC, Wolfagang H, Irizarry RA, Dudoit S. New York, NY: Springer Science + Business Media, Inc; 2005:397-420.

25. Benjamini $Y$, Hochberg $Y$ : Controlling the false discovery rate: a practical and powerful approach to multiple testing. J R Stat Soc Series B Stat Methodol 1995, 57:289-300.

26. Ashburner M, Ball CA, Blake JA, Botstein D, Butler H, Cherry JM, Davis AP, Dolinski K, Dwight SS, Eppig JT, et al: Gene ontology: tool for the unification of biology. The Gene Ontology Consortium. Nat Genet 2000, 25:25-29.

27. Huang DW, Sherman BT, Lempicki RA: Systematic and integrative analysis of large gene lists using DAVID bioinformatics resources. Nat Protoc 2008, 4:44-57.

28. Lourenço AP, Mackert A, Cristino AS, Simões ZLP: Validation of reference genes for gene expression studies in the honey bee, Apis mellifera, by quantitative real-time RT-PCR. Apidologie 2008, 39:372-385.

29. Hellemans J, Mortier G, De Paepe A, Speleman F, Vandesompele J: qBase relative quantification framework and software for management and automated analysis of real-time quantitative PCR data. Genome Biol 2007, 8:R19.

30. Maclsaac K, Fraenkel E: Practical strategies for discovering regulatory DNA sequence motifs. PLoS Comput Biol 2006, 2:e36.

31. Barchuk A, Cristino A, Kucharski R, Costa L, Simões Z, Maleszka R: Molecular determinants of caste differentiation in the highly eusocial honeybee Apis mellifera. BMC Dev Biol 2007, 7:70.

32. Cristino AS, Nunes FMF, Lobo CH, Bitondi MMG, Simões ZLP, Fontoura Costa L, Lattorff HMG, Moritz RFA, Evans JD, Hartfelder K: Caste development and reproduction: a genome-wide analysis of hallmarks of insect eusociality. Insect Mol Biol 2006, 15:703-714.

33. Roth F, Hughes J, Estep P, Church G: Finding DNA regulatory motifs within unaligned noncoding sequences clustered by whole-genome mRNA quantitation. Nat Biotech 1998, 16:939-945.

34. Bailey T, Elkan C: Unsupervised learning of multiple motifs in biopolymers using expectation maximization. Mach Learn 1995, 21:51-80.

35. Liu S, Brutlag D, Liu J: An algorithm for finding protein-DNA binding sites with applications to chromatin-immunoprecipitation microarray experiments. Nat Biotechnol 2002, 20:835-839.

36. Hughes J, Estep P, Tavazoie S, Church G: Computational identification of Cis-regulatory elements associated with groups of functionally related genes in Saccharomyces cerevisiae. J Mol Biol 2000, 296:1205-1214. 
37. Clarke ND, Granek JA: Rank order metrics for quantifying the association of sequence features with gene regulation. Bioinformatics 2003, 19:212-218.

38. Wingender $E$, Chen $X$, Hehl R, Karas H, Liebich I, Matys V, Meinhardt T, Prüß M, Reuter I, Schacherer F: TRANSFAC: an integrated system for gene expression regulation. Nucleic Acids Res 2000, 28:316-319.

39. Costa LF, Rodrigues FA, Cristino AS: Complex networks: the key to systems biology. Genet Mol Biol 2008, 31:591-601.

40. Ueda H, Sun GC, Murata T, Hirose S: A novel DNA-binding motif abuts the zinc finger domain of insect nuclear hormone receptor FTZ-F1 and mouse embryonal long terminal repeat-binding protein. $\mathrm{Mol} \mathrm{Cell} \mathrm{BiO}$ 1992, 12:5667-5672

41. Pinto LZ, Hartfelder K, Bitondi MM, Simões ZL: Ecdysteroid titers in pupae of highly social bees relate to distinct modes of caste development. J Insect Physiol 2002, 48:783-790.

42. Rutherford K, Parkhill J, Crook J, Horsnell T, Rice P, Rajandream MA, Barrell B: Artemis: sequence visualization and annotation. Bioinformatics 2000, 16:944-945.

43. Micas AFD: Proteínas do tegumento de abelhas Apis mellifera em metamorfose: identificação por espectrometria de massa. Tese. Ribeirão Preto, SP, Brazil: Universidade de São Paulo, Departamento de Genética; 2012.

44. Zhou X, Riddiford LM: Broad specifies pupal development and mediates the 'status quo' action of juvenile hormone on the pupal-adult transformation in Drosophila and Manduca. Dev 2002, 129:2259-2269.

45. Charles JP: The regulation of expression of insect cuticle protein genes. Insect Biochem Mol Biol 2010, 40:205-213.

46. Riddiford LM: Hormone action at the cellular level. In Comprehensive Insect Physiology, Biochemistry and Pharmacology. Volume 8. Edited by Kerkut GA, Gilbert LI. Oxford: Pergamon; 1985:2-84.

47. Moussian B: Recent advances in understanding mechanisms of insect cuticle differentiation. Insect Biochem Mol Biol 2010, 40:363-375.

48. Andersen SO, Rafn K, Roepstorff P: Sequence studies of proteins from larval and pupal cuticle of the yellow meal worm, Tenebrio molitor. Insect Biochem Mol Biol 1997, 27:121-131.

49. Guan X, Middlebrooks BW, Alexander S, Wasserman SA: Mutation of TweedleD, a member of an unconventional cuticle protein family, alters body shape in Drosophila. Proc Natl Acad Sci USA 2006, 103:16794-16799.

50. He N, Botelho JMC, McNall RJ, Belozerov V, Augustine Dunn W, Mize T, Orlando R, Willis JH: Proteomic analysis of cast cuticles from Anopheles gambiae by tandem mass spectrometry. Insect Biochem Mol Biol 2007, 37:135-146.

51. Jasrapuria S, Arakane Y, Osman G, Kramer KJ, Beeman RW, Muthukrishnan S: Genes encoding proteins with peritrophin A-type chitin-binding domains in Tribolium castaneum are grouped into three distinct families based on phylogeny, expression and function. Insect Biochem Mol Biol 2010, 40:214-227.

52. Andersen SO: Studies on proteins in post-ecdysial nymphal cuticle of locust, Locusta migratoria, and cockroach, Blaberus craniifer. Insect Biochem Mol Biol 2000, 30:569-577.

53. Lourenço AP, Zufelato MS, Bitondi MMG, Simões ZLP: Molecular characterization of a CDNA encoding prophenoloxidase and its expression in Apis mellifera. Insect Biochem Mol Biol 2005, 35:541-552.

54. Elias-Neto M, Soares MPM, Simões ZL, Hartfelder K, Bitondi MM: Developmental characterization, function and regulation of a Laccase2 encoding gene in the honey bee, Apis mellifera (Hymenoptera, Apinae). Insect Biochem Mol Biol 2010, 40:241-251.

55. Locke M: The localization of a peroxidase associated with hard cuticle formation in an insect, Calpodes ethlius stoll, Lepidoptera, Hesperiidae. Tissue Cell 1969, 1:555-574.

56. Hasson C, Sugumaran M: Protein cross-linking by peroxidase - possible mechanism for sclerotization of insect cuticle. Arch Insect Biochem Physiol 1987, 5:13-28.

57. Bae Y-A, Cai G-B, Kim S-H, Zo Y-G, Kong Y: Modular evolution of glutathione peroxidase genes in association with different biochemical properties of their encoded proteins in invertebrate animals. BMC Evol Biol 2009, 9:72

58. Davies KG, Curtis RHC: Cuticle surface coat of plant-parasitic nematodes. Annu Rev Phytopathol 2011, 49:135-156.

59. Butler MJ, Jacobsen TL, Cain DM, Jarman MG, Hubank M, Whittle JRS, Phillips $R$, Simcox A: Discovery of genes with highly restricted expression patterns in the Drosophila wing disc using DNA oligonucleotide microarrays. Dev 2003, 130:659-670.
60. Hayes SA, Miller JM, Hoshizaki DK: Serpent, a GATA-like transcription factor gene, induces fat-cell development in Drosophila melanogaster. Dev 2001, 128:1193-1200.

61. Shah AP, Nongthomba U, Kelly Tanaka KK, Denton MLB, Meadows SM Bancroft N, Molina MR, Cripps RM: Cardiac remodeling in Drosophila arises from changes in actin gene expression and from a contribution of lymph gland-like cells to the heart musculature. Mech Dev 2011, 128:222-233.

62. Bour BA, O'Brien MA, Lockwood WL, Goldstein ES, Bodmer R, Taghert PH, Abmayr SM, Nguyen HT: Drosophila MEF2, a transcription factor that is essential for myogenesis. Genes Dev 1995, 9:730-741.

63. LaJeunesse $D$, Shearn A: $E(z)$ : a polycomb group gene or a trithorax group gene? Dev 1996, 122:2189-2197.

64. Pirrotta V, Bickel S, Mariani C: Developmental expression of the Drosophila zeste gene and localization of zeste protein on polytene chromosomes. Genes Dev 1988, 2:1839-1850.

65. Gomez-Skarmeta JL, Campuzano S, Modolell J: Half a century of neural prepatterning: the story of a few bristles and many genes. Nat Rev Neurosci 2003, 4:587-598.

66. Schweizer L, Nellen D, Basler K: Requirement for Pangolin/dTCF in Drosophila Wingless signaling. Proc Natl Acad Sci USA 2003, 100:5846-5851.

67. Zeng YA, Rahnama M, Wang S, Lee W, Verheyen EM: Inhibition of Drosophila Wg signaling involves competition between Mad and Armadillo/ $\beta$-Catenin for dTcf binding. PLOS ONE 2008, 3:e3893.

68. Wang $\mathrm{H}-\mathrm{B}$, Iwanaga $\mathrm{M}$, Kawasaki H: Activation of BMWCP10 promoter and regulation by BR-C Z2 in wing disc of Bombyx mori. Insect Biochem $\mathrm{Mol}$ Biol 2009, 39:615-623.

69. Wang H-B, Nita M, Iwanaga M, Kawasaki H: $\beta F T Z-F 1$ and Broad-Complex positively regulate the transcription of the wing cuticle protein gene, BMWCP5, in wing discs of Bombyx mori. Insect Biochem Mol Biol 2009, 39:624-633.

70. Sandstrom DJ, Restifo LL: Epidermal tendon cells require Broad Complex function for correct attachment of the indirect flight muscles in Drosophila melanogaster. J Cell Sci 1999, 112:4051-4065.

71. Vigoreaux JO, Swank DM: The development of the flight and leg muscle. In Comprehensive Molecular Insect Science. Volume 2. Edited by Gilbert L, latrou K, Gill S. Oxford: Elsevier Press; 2005:45-84.

72. Kozopas KM, Nusse R: Direct flight muscles in Drosophila develop from cells with characteristics of founders and depend on DWnt-2 for their correct patterning. Dev Biol 2002, 243:312-325.

73. Santos $A E$, Bitondi MMG, Simões ZLP: Hormone-dependent protein patterns in integument and cuticular pigmentation in Apis mellifera during pharate adult development. J Insect Physiol 2001, 47:1275-1282.

74. Zufelato MS, Bitondi MM, Simões ZL, Hartfelder K: The juvenile hormone analog pyriproxyfen affects ecdysteroid-dependent cuticle melanization and shifts the pupal ecdysteroid peak in the honey bee (Apis mellifera). Arthropod Struct Dev 2000, 29:111-119.

doi:10.1186/1471-2164-14-576

Cite this article as: Soares et al:: Genes involved in thoracic exoskeleton formation during the pupal-to-adult molt in a social insect model, Apis mellifera. BMC Genomics 2013 14:576.

\section{Submit your next manuscript to BioMed Central and take full advantage of:}

- Convenient online submission

- Thorough peer review

- No space constraints or color figure charges

- Immediate publication on acceptance

- Inclusion in PubMed, CAS, Scopus and Google Scholar

- Research which is freely available for redistribution 